Open Access

\title{
Theoretical and experimental optimization of DMT-based visible light communication under lighting constraints
}

\author{
Ahmad Jabban ${ }^{1 *}$, Sylvain Haese ${ }^{1}$ and Maryline Helard ${ }^{1}$
}

\section{${ }^{*}$ Correspondence:}

ahmad.jabban@insa-rennes.fr

'European University of Brittany, INSA, IETR, UMR 6164, F-35708,

Rennes, France

\begin{abstract}
High spectral efficiency is a key element that drives research for future wireless communication systems in order to meet the increasing demand for ubiquitous connectivity despite a limited radio frequency spectrum. Visible light communications (VLC) that allow exploiting the existing infrastructure for both lighting and communications could be an effective and economic solution. Thanks to the revolution in the field of solid-state lighting and the accelerated development of light-emitting diodes (LED), VLC becomes one of the most promising new technologies for the next generation of wireless communication systems. However, the limited bandwidth of the LEDs remains a major challenge that is limiting VLC from achieving very high data rates. In this paper, we propose to adapt the discrete multi-tone (DMT) modulation scheme of indoor VLC communications for data rate enhancement. With the help of bit and power-loading algorithms, a transmission bandwidth much larger than the 3-dB bandwidth of the LED could be exploited. We firstly present the typical lighting requirements for indoor applications and investigate the maximal bit-rate of DMT-based VLC. Then, with a regular luminance level for normal office work, an overall optimization of DMT configuration scheme in terms of modulated bandwidth, subcarrier number, cyclic prefix length, and clipping level is proposed. Theoretical analysis and verifications by experiments are simultaneously carried out. Based on the proposed configuration, we demonstrate a 100-Mbps VLC wireless transmission employing low-cost components under realistic lighting constraints.
\end{abstract}

Keywords: Bit-loading, Channel capacity, Discrete multi-tone, Light-emitting diode, System optimization, Visible light communication

\section{Introduction}

Visible light communication (VLC) is a technology that provides an opportunity for highspeed low-cost wireless communications. Due to their distinct advantages such as low deployment cost, license-free application, wide bandwidth, energy efficiency, high security, and immunity to interference from electromagnetic sources, VLC is one of the most promising new technologies for the next generation of wireless communication systems [1]. For indoor applications, intensity modulation and direct detection (IM/DD) is often

(C) The Author(s). 2020 Open Access This article is licensed under a Creative Commons Attribution 4.0 International License which permits use, sharing, adaptation, distribution and reproduction in any medium or format, as long as you give appropriate credit to the original author(s) and the source, provide a link to the Creative Commons licence, and indicate if changes were made. The images or other third party material in this article are included in the article's Creative Commons licence, unless indicated otherwise in a credit line to the material. If material is not included in the article's Creative Commons licence and your intended use is not permitted by statutory regulation or exceeds the permitted use, you will need to obtain permission directly from the copyright holder. To view a copy of this licence, visit http://creativecommons.org/licenses/by/4.0/. 
used as a practical transmission scheme to achieve simple and low-cost modulation and demodulation. This means that only the signal intensity is modulated and there is no phase information. At the transmitter side, the emitted wave is real-valued and unipolar (positive-valued), and the signal is directly modulated onto the instantaneous power of the optical carrier. At the receiver side, a photo-detector (PD) produces a current proportional to the received instantaneous power, enabling the implementation of simple low-cost transceiver devices without the need for complex high-frequency circuit designs [2].

Due to the revolution in the field of solid-state lighting, efficient and inexpensive illumination light-emitting diodes (LED) are spreading for illumination purposes. Thanks to their energy-efficiency and longevity compared to the traditional lighting systems, they are expected to replace the conventional incandescent and fluorescent lamps. Such sources can be switched and modulated at high speed without being detected by the human eye, offering the possibility of exploiting the illumination levels within a room in parallel with high-data rate wireless communications [3, 4]. Therefore, the ceiling white light used for illumination in an office can be used to transmit data to a receiver placed on a desk. Typical white light fixtures achieve more than 400 lux to provide sufficient indoor illumination, and this lighting level is enough to transmit data at high signal-tonoise ratio (SNR) [5]. In general, there are several ways to produce white light by the LEDs with different impacts on the optical bandwidth. In a first method, white light is produced by three or four color LED chips. Typical red, green, and blue (tri-chromatic mode) or red, green, blue, and yellow (tetra-chromatic mode) are combined to provide the desired light color. In a second way (dichromatic mode), a single blue LED coated with a yellowish phosphor layer which uses the phosphor to convert the light emitted by the blue LED to other regions of the spectrum and produce the white light $[1,5]$. The trichromatic and tetra-chromatic approaches allow different data to be sent on each color, with a bandwidth of about $15 \mathrm{MHz}$ available for each LED chip. However, maintaining color balance can be challenging and the devices become more complex and expensive compared to dichromatic mode. Moreover, multi-color LED chips suffer from color shifts due to aging and different junction temperatures of the LEDs [6]. Therefore, LEDs coated by phosphor layer are most widely used for lighting applications owing to their lower cost, an easy construction and a simplest electrical control. Nevertheless, they provide a smaller bandwidth than multicolor LEDs. This is a major challenge for high-data rate communications using these devices. Using a blue filter before the photo-detector at the receiver side allows eliminating the slow-response yellow light component and increasing the LED modulation bandwidth to the range of $10-20 \mathrm{MHz}$. But this is at the cost of significant degradation of the received power [7].

High spectrally efficient modulation format is an important approach to deal with the bandwidth problem. Among modulation formats, discrete multi-tone (DMT) is a popular choice since adaptive bit and power-loading techniques on each subcarrier as well as simple equalization can be used to highly exploit the available bandwidth of the LED and to increase the capacity under limited bandwidth [8]. The number of orthogonal subcarriers is chosen so that the bandwidth of the modulated signal is smaller than the coherence bandwidth of the optical channel, and consequently, each sub-channel can be considered as a flat-fading channel [9]. A cyclic prefix (CP) of length larger than the channel delay spread can also be introduced after inverse fast Fourier transform (IFFT) to deal with 
the inter symbol interference (ISI). Nevertheless, DMT has a high peak-to-average-power ratio (PAPR) due to the summation over a large number of subcarriers. The high PAPR or dynamic range of DMT makes it very sensitive to nonlinear distortions of LEDs. To counteract the LED nonlinearity problem, it is necessary to operate the LED in a small current range where its output characteristics are linear enough or to pre-distort the signal such that the output of the LED has the desired shape. Moreover, to reduce the PAPR, a clipping technique could be applied for signals that exceed a desired level. However, more clipping noise is introduced with the high-clipped signal. Therefore, in order to enhance the DMT transmission over a VLC channel, a global optimization of system parameters must be realized.

In the literature, many works have used DMT modulation for VLC [8, 10-20]. Some works have analyzed the blue filter effectiveness on the transmission rates $[8,10,11]$. The authors in those works concluded that the optical blue filter does not enhance the white light LED "practical" modulation bandwidth in the case of DMT modulation and even significantly degrades the transmission performance. Other works reach the maximization of the transmission data rate using advanced components and materials [12-17]. The authors of [12] used a phosphorescent white light LED luminary with improved factory designed spherical lens to achieve a 100-Mbps data rate and then 513 Mbps by using low-noise avalanche photo-diode and advanced circuits to enhance the 3-dB LED bandwidth up to $35 \mathrm{MHz}$ [13]. The same authors demonstrated a data rate of $803 \mathrm{Mbps}$ [14] by using three channels having similar frequency characteristics with 3$\mathrm{dB}$ points around $15-20 \mathrm{MHz}$ in a single red-green-blue (RGB) LED luminary and then 1.25 Gbps [15] by improving the integrated 3-channel RGB LED driver. The achieved data rates are presented at 1000 lux illumination level and 10-12 cm between the emitter and the receiver. High transmission rates of 1 Gbps with phosphorescent LED [16] and 3.4 Gbps with RGB LED [17] are mentioned with the help of advanced circuits allowing respectively $180 \mathrm{MHz}$ and $280 \mathrm{MHz}$ modulation bandwidths. Other modulation schemes based on DMT were proposed in [18-20]. Dimming technique is applied in [18] to provide color control in the red-green-blue LEDs and $103.5 \mathrm{Mb} / \mathrm{s}(3 \times 34.5 \mathrm{Mbps})$ speed is demonstrated using RGB LEDs. In [19], DMT transmission is proposed to simultaneously provide positioning and high-data rate communication, and in [20], a transmission rate of 2.1 Gbps is achieved with wavelength-division multiplexing-based DMT transmission using a red-green-blue-amber (RGBA) LED model. Nevertheless, the authors of these papers reported their results without presenting analytical description or optimization of DMT transmission. None of them indicated the relationship between, luminance level, subcarrier number, cyclic prefix length, and clipping level with the maximal data rate. To enhance the transmission over VLC, an overall optimization of system parameters should be performed as well as a mixed theoretical experimental analysis for system optimization should be considered for any type of components and materials. Moreover, the system capacity with bit- and power-loading is directly related to the modulated bandwidth [21,22]. A detailed study should be realized to determine the optimal modulated bandwidth suitable for each desired luminance level allowing the maximal transmission rate. Otherwise, we would like to report that very high data rates are mentioned in the literature using more complex types of lighting sources such as micro-LEDs [23-25] and special made LEDs including organic [26] semiconductor [27] and photonic crystal materials [28]. A maximum data rate of $8 \mathrm{Gbps}$ is achieved in [25]. Moreover, vertical-cavity 
surface-emitting laser (VCSEL) is also used as lighting source device in $[29,30]$ allowing data rate of $11.1 \mathrm{Gbps}$ in [29] and enhanced data rate of 25 Gbps [30] by using two-stage external-injected VCSEL. Although several billions of bits per second are reported using these devices, but there are many problems in practical applications. Firstly, these devices have more complicated production methods, minimal luminous power, and higher costs compared to commercial white LEDs. Moreover, they are mainly designed for increasing the data rate in a communication system, and it is difficult to take into account other functions as the illumination requirements at the same time [31].

In this paper, we propose a global optimization of DMT transmissions considering commercial phosphor white LEDs used for both illumination and communication. This optimization aims to maximize the transmission bit rate in practical VLC systems employing low-cost components. The main contributions of this paper are summarized as follows: we firstly investigate the transmission bit rate of DMT modulation with bit-loading according to different luminance levels taking into account all lighting and communication system parameters. To the best of our knowledge, this is the first time this overall investigation in VLC is explicitly analyzed. Then, we propose a theoretical low-pass channel model similar to the real frequency response of VLC link. The proposed channel model is employed for the theoretical analysis and optimization. Next, for typical indoor lighting requirements, we determine the optimal values of subcarrier number, modulated bandwidth, cyclic prefix length, and clipping level allowing for the maximal bit rate. The selection of these parameters is made with the help of theoretical and experimental analysis. We believe that this is the first work that shows a mixed theoretical-experimental optimization of DMT parameters in VLC. Based on the optimized configuration, we finally demonstrate a $100 \mathrm{Mbps}$ low-cost component VLC wireless transmission system under lighting constraints.

The rest of this paper is structured as follows. Section 2 provides an analytical description of lighting and optical communication properties of an indoor VLC system. Section 3 describes the discrete multi-tone modulation over VLC channel and introduces the maximal bit rate with bit-loading according to different luminance levels. In Section 4, we present our experimental setup and show in Section 5, the measured parameters of system required for optimization. Section 6 outlines the theoretical optimization and Section 7 shows the experimental optimization results to validate the optimal setups. Based on the proposed configuration, we demonstrate in Section 8 a 100-Mbps wireless VLC system with low-cost components. Finally, concluding remarks and future work details are given in Section 9.

\section{Lighting and optical communication analysis of VLC for indoor applications}

In this section, we present an analytical description of typical indoor lighting requirements as well as the basic lighting and optical communication properties of phosphorbased white-light LEDs. The lighting level and the optical power at the receiver side are also introduced.

\subsection{Typical indoor lighting requirements}

In general, LED lights have two basic lighting properties, the luminous flux given in lumen $(\mathrm{lm})$ and the luminance of light given in lux. Luminous flux is a quantitative expression of the brilliance of a visible light source. On the other hand, the luminance of light is defined 
as the incident luminous flux per unit area and expresses the brightness of an illuminated surface. In general, the luminous flux of a single LED can be defined as following [32]:

$$
\Phi_{\text {lum }}=K_{m} \int_{380}^{780} V(\lambda) P(\lambda) d \lambda \quad[\operatorname{lm}]
$$

where $K_{m}$ (lumen per watt) is the maximum luminous efficacy, which is $683 \mathrm{~lm} / \mathrm{W}$ at a 555-nm wavelength, $V(\lambda)$ is the standard luminosity curve versus wavelength $\lambda$, and $P(\lambda)$ is the source spectral radiant power density function, which represents the amount of source power according to each wavelength in the electromagnetic spectrum.

Assuming that LED lighting has a Lambertian radiation pattern, the horizontal luminance at a point of the receiver surface can be expressed as a function of luminous flux by $[33,34]$ :

$$
E_{h}=\frac{(m+1) \cos ^{m}(\phi) \cos (\psi)}{2 \pi r^{2}} \Phi_{\text {lum }} \quad[\text { lux }]
$$

where $\phi$ is the angle of irradiance with respect to the axis normal to the transmitter surface, $\psi$ the angle of incidence with respect to the axis normal to the receiver surface, $r$ the distance between the LED and the illuminated surface, and $m=\frac{-\ln 2}{\ln \left(\cos \left(\Phi_{1 / 2}\right)\right.}$ expressing directivity of the source beam with $\Phi_{1 / 2}$ the semiangle at half luminance of a LED. For this study, we used a single LUXEON 3020 LED for which, as according to the data sheet (L130-3080002011001), $\Phi_{1 / 2}$ equals to $55^{\circ}$ [35]. The luminance of light, in the LED axis, emitted by this LED biased at $120 \mathrm{~mA}$, is given as a function of the distance in Fig. 1 .

The luminance of light in indoor applications is governed by various standardization bodies. Table 2.1 in [36] provides typical luminance values for different applications based

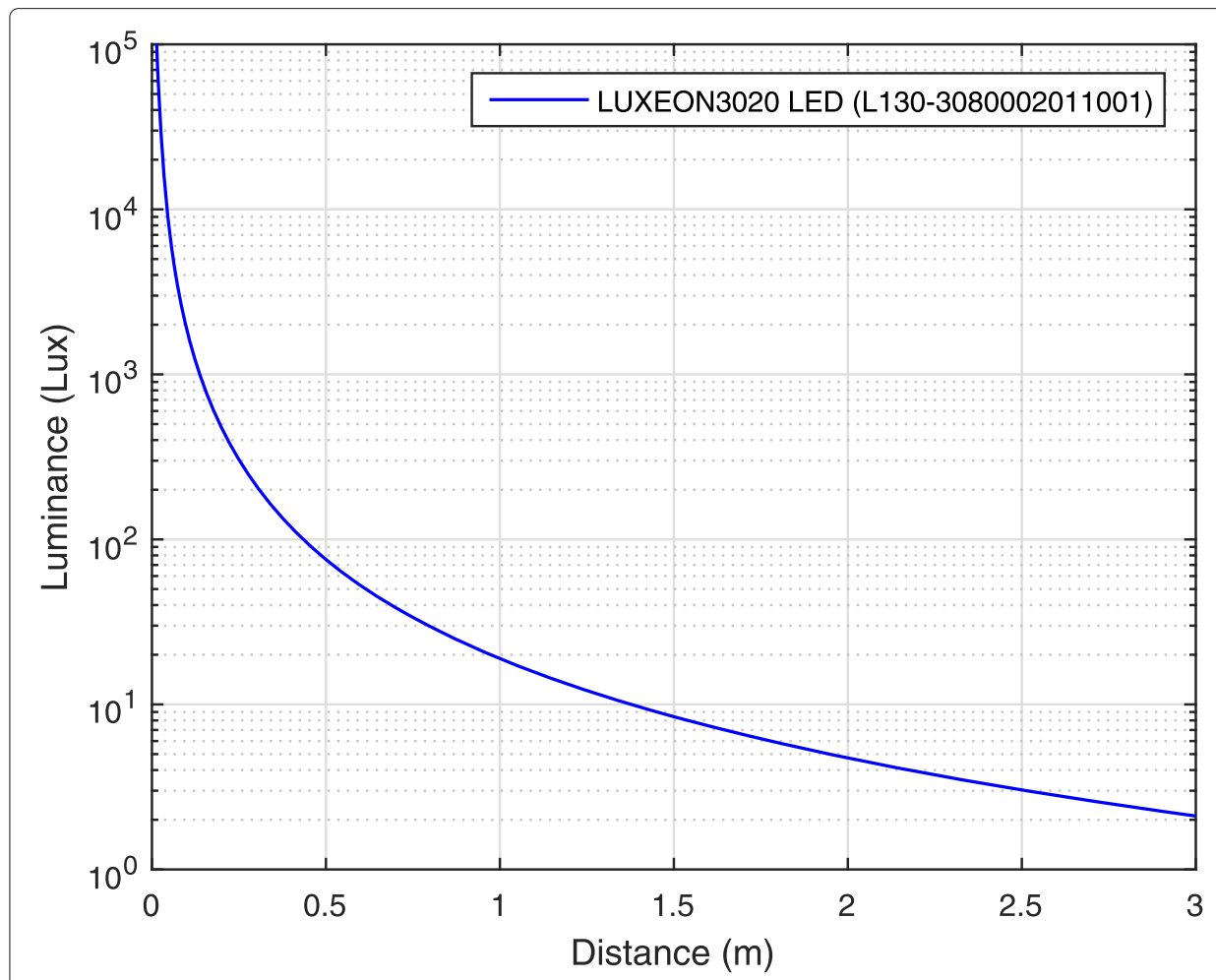

Fig. 1 Luminance from a single LED biased at $120 \mathrm{~mA}$ according to the distance with the illuminated surface 
on Deutsche Institut fur Normung (DIN) standard [37]. By this set of standards, luminance of 200 to 1000 lux is required for regular rooms with typical 500 lux for a normal office work. From Fig. 1, we can see that a single LED can only provide limited luminous flux and only over a limited area. Therefore, to illuminate a much larger environment such as work offices, arrays of multi-LEDs organized in luminary form would be needed. This system ensures the sufficient illumination levels and at the same time could offer spatial diversity to avoid blocking. The total luminance at the work surface in the case of using array of LEDs is equal to the sum of the luminance of each LED in the luminary:

$$
E_{h}=(m+1) \sum_{i=1}^{N_{L E D}} \frac{\cos ^{m}\left(\phi_{i}\right) \cos \left(\psi_{i}\right)}{2 \pi r_{i}^{2}} \Phi_{i, l u m}
$$

where $N_{L E D}$ is the number of LEDs for a given scenario.

For a classical luminary in a typical room of a 3-m height, LEDs are separated by $1 \mathrm{~cm}$ and the distance between the luminary and a receiver situated on a task area for working purposes, typically $0.75 \mathrm{~m}$ above the floor, is about $2.25 \mathrm{~m}$. In this case, the difference in path lengths from the receiver to each LED is $0.22 \mathrm{~cm}$, causing propagation time differences equal to 7.33 ps. Moreover, the difference in channel gains between LEDs is differing on the order of $0.41 \%$ between different LEDs. Consequently, all LEDs in each luminary could be assumed to have identical propagation delays and gains to the receiver [38]. Accordingly, the required luminous flux and number of LUXEON3020 LEDs in a luminary for a minimum luminance of 500 lux in the task area are respectively $7078 \mathrm{~lm}$ and 134 LEDs. The luminance level on the task area according to the number of LEDs is shown in Fig. 2.

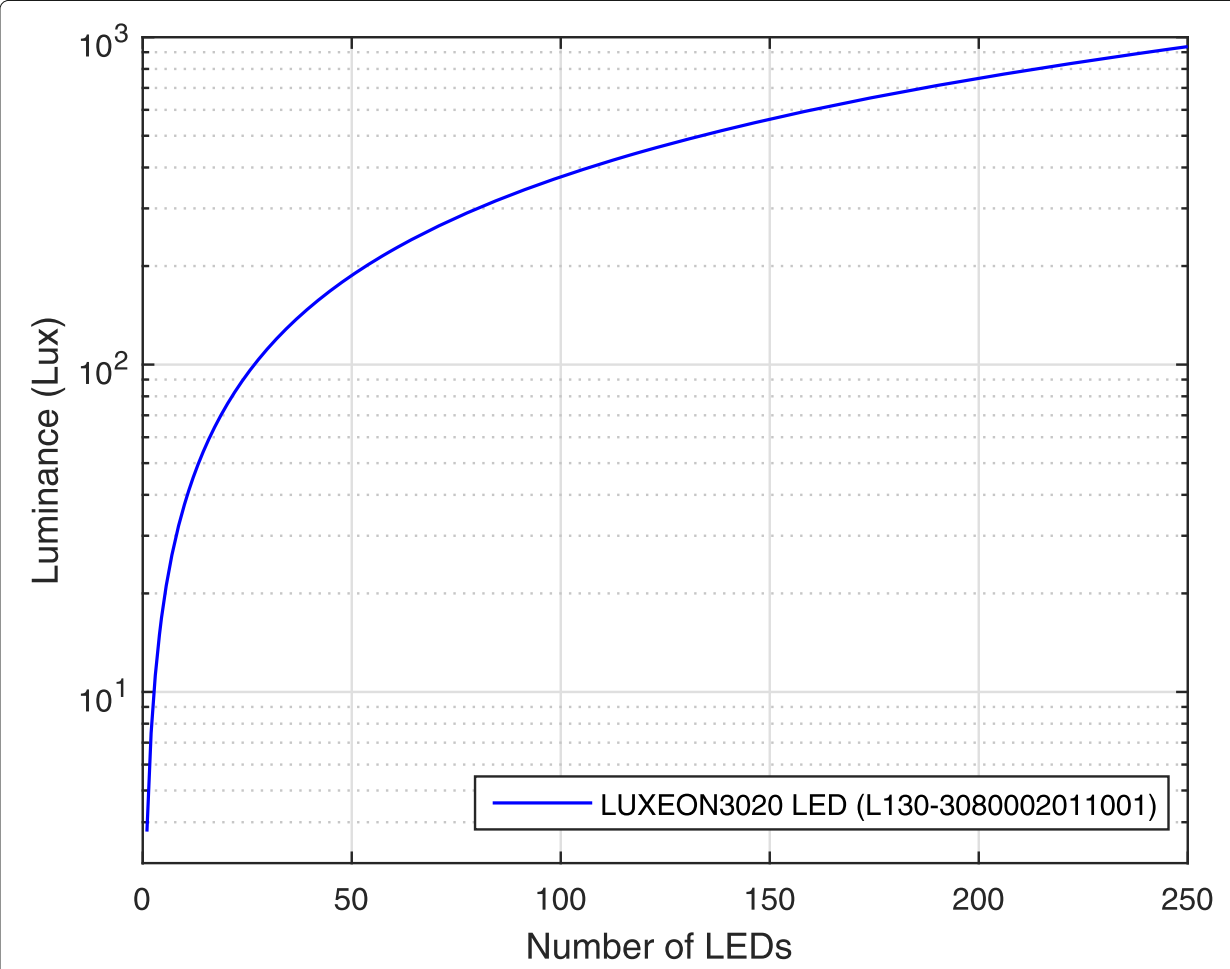

Fig. 2 Luminance at $2.25 \mathrm{~m}$ right below the luminary. The luminance level on the task area according to the number of LEDs 


\subsection{Optical power analysis}

Since LEDs in indoor VLC systems can be used for both illumination and communication, it is necessary to define the transmitted optical power $P_{\text {opt }}$ which indicates the total energy radiated from the LED. As stated in [32], the radiated optical power of a single LED can be calculated by:

$$
P_{\text {opt }}=\int_{\lambda_{\min (380)}}^{\lambda_{\max (780)}} P(\lambda) d \lambda \quad[W]
$$

and an optical luminous efficiency which describes the conversion efficiency from the LED optical power to luminosity is classically given by:

$$
\eta=\frac{\Phi_{\text {lum }}}{P_{\text {opt }}} \quad[\operatorname{lm} / W]
$$

At the transmitter side, the radiated optical power from a LED is an important parameter for wireless transmission. However, most data sheets of white LEDs only provide luminous flux in lumen, which is a useful metric for illumination design. Therefore, measurements are conducted to determine the transmitted optical power in watts for the considered optical source [39]. In our study, we use a LUXEON3020 LED with a luminous flux of $53 \mathrm{~lm}$. This LED operates at $120 \mathrm{~mA}$ bias current and dissipates $366 \mathrm{~mW}$ of electrical power. Using the spectral radiant power density function of the LED given in the data sheet [35], the CIE 1978 (International Commission on Illumination) eye sensitivity function in the photonic vision regime [40], Eqs. (1) and (4), the average output optical power of the LED is calculated to be approximately $171 \mathrm{~mW}$. Thanks to Eq. (5), the optical conversion factor is therefore approximated to $\eta=310 \mathrm{~lm} / \mathrm{W}$.

At the receiver side, two components of optical power could be received by the photodetector, the signal power received directly from the LEDs and the signal power that comes from the reflections of light onto objects, and these reflections may arrive at different times with different attenuations. In general, the optical power associated with two or more reflections is relatively small and can be neglected [32]. Considering the reflection onto the walls, the total received optical power at the receiver is given by:

$$
P_{r}=\sum^{N_{L E D}}\left\{P_{r_{-} L O S}+\int_{\text {Walls }} P_{r_{-} N L O S}\right\}
$$

The received optical power from a single LED at the receiver in the case of one direct light path $\left(P_{r_{-} L O S}\right)$ and one reflection $\left(P_{r_{-} N L O S}\right)$ are given as by [32]:

$$
\begin{aligned}
& P_{r_{-} L O S}=P_{\text {opt }} \frac{(m+1) A_{r}}{2 \pi r^{2}} \cos ^{m}(\phi) T_{s}(\psi) g(\psi) \cos (\psi) \quad 0 \leqslant \psi \leqslant \psi_{c} \\
& P_{r_{-} N L O S}=P_{\text {opt }} \sum_{j} \frac{A_{r}(m+1) \rho_{j} \Delta A_{w}}{2 \pi^{2} d_{s j}^{2} d_{r j}^{2}} \cos ^{m}\left(\phi_{s j}\right) . \\
& \cos \left(\alpha_{s j}\right) \cos \left(\beta_{r j}\right) \cos \left(\psi_{r j}\right) T_{s}(\psi) g(\psi)
\end{aligned}
$$

where $P_{\text {opt }}$ is the optical power emitted by a single LED, $A_{r}$ the effective collection area of the detector, $T_{s}(\psi)$ the filter transmission gain, $g(\psi)$ the optical concentrator gain, $\Delta A_{w}$ the area of reflecting elements, $\phi_{s j}$ is the angle of irradiance to the transmitter surface, $\alpha_{s j}$ the angle of irradiance to a reflective element $j, \beta_{r j}$ the angle of irradiance to a receiver, $\psi_{r j}$ the angle of incidence from the reflective element $j, d_{s j}$ the distance from $s$ to $j$, and $\rho_{j}$ 
is the reflection coefficient of $j$. Typical measured values of $\rho$, for different wall materials, are available in [41].

Generally, when both the direct and reflected components are incident on a receiver, the direct component significantly presents higher intensity [42]. For a luminary situated in the center of a typical room to illuminate a task area right below the luminary for a minimum luminance of 500 lux, and supposing that the arrival times of the reflections are less than the symbol duration, only $1.59 \%$ of the total received optical power is received from reflections.

\section{Discrete multi-tone modulation in VLC}

\subsection{DMT transmission in indoor VLC systems}

Discrete multi-tone is one of modulation schemes employed in VLC in order to extend the transmission bandwidth and exploit the high level of SNR in indoor applications [5]. A schematic diagram of an indoor VLC system using DMT modulation is shown in Fig. 3.

Information is firstly coded and mapped to quadrature amplitude modulation (QAM) symbols and modulated onto $N$ orthogonal subcarriers located in the considered frequency band. It should be noticed that in IM/DD VLC transmissions, the electrical signal modulated onto the instantaneous power of the LED should be real-valued and unipolar (positive-valued). The real-valued DMT symbols are obtained by the use of Hermitian symmetry and a $2 \mathrm{~N}$-point IFFT. The real-valued time domain signal $S(k)$ at the output of the IFFT block is given as follows [43]:

$$
\begin{gathered}
S(k)=\frac{1}{\sqrt{2 N}} \sum_{n=0}^{2 N-1} 2 \cdot \operatorname{Re}\left\{X_{n} \cdot \exp \left(j 2 \pi n \frac{k}{2 N}\right)\right\} ; k=0,1, \cdots, 2 N-1 \\
X_{0}=X_{N}=0 \text { and } X_{2 N-n}=X_{n}^{*}
\end{gathered}
$$

where $X_{n}^{*}$ denotes the complex conjugate operation of sample $X_{n}$.

A cyclic prefix of length $L_{C P}$ designed larger than the channel delay spread is then added to the start of each DMT symbol in order to deal with the inter symbol interference:

$$
C P=\left[S\left(2 N-L_{C P}\right) \cdots S(2 N-1)\right]
$$

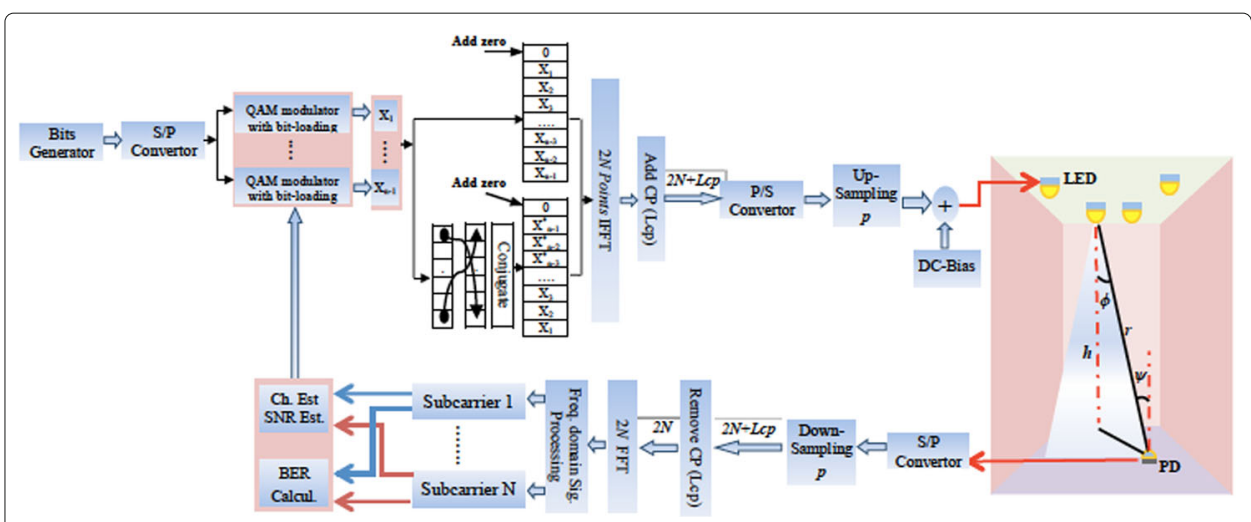

Fig. 3 Schematic diagram of an indoor VLC system using DMT modulation 
Before the conversion of the electrical signal to an optical signal, a positive direct current (DC) bias is introduced to the bipolar signal in the time domain for unipolar signal generation and clipping is applied to shape the signal with the desired amplitude range.

\subsection{Capacity and bit rate in DMT with bit-loading}

The channel capacity in a communication system is directly related to the SNR level and the used bandwidth by:

$$
C=W \cdot \log _{2}(1+S N R)
$$

where $S N R=\frac{P}{N_{0} W}$ and $P$ is the signal power, $N_{0}$ the noise power spectral density, and $W$ is the used bandwidth [44].

In DMT transmission, bit-loading algorithm is usually carried out in order to optimize the transmission bit rate $[45,46]$. Each orthogonal sub-channel can be independently treated and the number of bits allocated to each subcarrier is made according to its SNR value. Thus, with optimum QAM constellation sizes allocated to different subcarriers, it is possible to closely reach the maximum available bit rate. In DMT, the total time duration of one symbol can be expressed as $T_{D M T}=\frac{N}{B}+\frac{N_{C P}}{B}$ where $B$ is the occupied bandwidth, $N$ the number of subcarriers, and $N_{C P}=L_{C P} / 2$. Moreover, the capacity $C_{D M T}$ and the maximal bit-rate $D_{\max }$ in terms of electrical SNR can be given as follows:

$$
\begin{gathered}
C_{D M T}=\frac{B}{N+N_{C P}} \sum_{i=1}^{N} \operatorname{round}\left[\log _{2}\left(1+S N R_{e}(i)\right)\right] \\
D_{\text {max }}=\frac{B}{N+N_{C P}} \sum_{i=1}^{N} \text { round }\left[\log _{2}\left(1+\frac{S N R_{e}(i)}{\Gamma}\right)\right]
\end{gathered}
$$

where $S N R_{e}(i)$ is the electrical SNR at the $i$ th subcarrier and $\Gamma$ is the gap between the SNR required to achieve Shannon capacity and that necessary to achieve this capacity at a given bit error rate [45]. In case of QAM, $\Gamma$ with a target symbol error rate (SER) can be represented as follows [43]:

$$
\Gamma=\frac{1}{3}\left[Q^{-1}\left(\frac{S E R}{4}\right)\right]^{2}
$$

In DMT modulation with bit-loading, a transmission bandwidth much larger than the 3-dB bandwidth of the LED, could be exploited and consequently the LED frequency characteristic becomes a crucial key to calculate the channel capacity. According to the optical power distribution in the work area, the corresponding $S N R_{e}$ at the $i$ th subcarrier can be expressed as [32]:

$$
\operatorname{SNR}_{e}(i)=\frac{\left(R_{i} P_{i}\left|H_{i}\right|\right)^{2}}{\sigma_{i}^{2}}
$$

where $R_{i},\left|H_{i}\right|, \sigma_{i}^{2}$, and $P_{i}$ are respectively the photo-detector responsivity, the LED magnitude response, the noise power, and the optical power at the $i$ th subcarrier. At the transmitter side, the allocated power to subcarriers can be optimized by water-filling algorithm [47]. In [45], it is indicated that equal power allocation and water-filling algorithm can reach similar performance. Since it is the simplest, equal power allocation is considered in our system. Supposing that the receive signal is dominated by the direct signal component and as all LEDs in each luminary could be assumed to have identical delays 
and gains to the receiver [38], $P_{i}$ can be calculated based on (6) and (7) as:

$$
P_{i}=N_{L E D} P_{r i \_} L O S=N_{L E D}\left(\frac{P_{o p t}}{N} \cdot \frac{(m+1) A_{r}}{2 \pi r^{2}} \cdot \cos ^{m}(\phi) T_{S}(\psi) g(\psi) \cos (\psi)\right)
$$

Moreover, using Eqs. (3) and (5), the total luminance at the work surface can be given by:

$$
E_{h}=(m+1) N_{L E D} \frac{\cos ^{m}(\phi) \cos (\psi)}{2 \pi r^{2}}\left(\eta P_{o p t}\right)
$$

From (17) and (18), the corresponding power at the $i$ th subcarrier can be calculated by:

$$
P_{i}=\frac{E_{h} A_{r} T_{s}(\psi) g(\psi)}{N \eta}
$$

Consequently, $S N R_{e}$ as function of the horizontal luminance can be estimated by:

$$
\operatorname{SNR}_{e}(i)=\frac{\left(R_{i}\left|H_{i}\right| A_{r} T_{s}(\psi) g(\psi) E_{h}\right)^{2}}{N^{2} \eta^{2} \sigma_{i}^{2}}
$$

Replacing $S N R_{e}$ from (20) in (13) and (14), we can calculate the channel capacity and the maximal bit rate according to the horizontal luminance as follows:

$$
\begin{aligned}
C_{D M T} & =\frac{B}{N+N_{C P}} \sum_{i=1}^{N} \log _{2}\left(1+\frac{\left(R_{i}\left|H_{i}\right| A_{r} T_{s}(\psi) g(\psi) E_{h}\right)^{2}}{N^{2} \eta^{2} \sigma_{i}^{2}}\right) \\
D_{\max } & =\frac{B}{N+N_{C P}} \sum_{i=1}^{N} \log _{2}\left(1+\frac{\left(R_{i}\left|H_{i}\right| A_{r} T_{s}(\psi) g(\psi) E_{h}\right)^{2}}{\Gamma N^{2} \eta^{2} \sigma_{i}^{2}}\right)
\end{aligned}
$$

From (21) and (22), we can observe that, for given materials and components, the parameters $R_{i}, A_{r}, T_{s}(\psi), g(\psi)$, and $\eta$ have constant values while other parameters as $B$, $N, N_{C P}, E_{h}$, and $\sigma_{i}$ are variable and the choice of their values has a direct impact on the channel capacity and the maximal bit rate. Moreover, it should be noticed that bit-loading technique is typically required in DMT transmission over indoor VLC channel to mitigate the LED frequency characteristic and circumvent their spectral effects. In the case of optical sources composed of a blue LED chip and a phosphor coated layer, the frequency response of the source is a combination of the frequency responses of the blue LED chip and that of coated phosphor. Based on [48] and the correction factors presented in [31], the frequency response of a blue LED coated by phosphor layer could be modeled as low-pass filter with a transfer function given as following:

$$
\left|H_{i}\right|=\exp \left[-\left(\frac{f_{i}}{f_{0}}\right)^{\frac{1}{2}}\right]
$$

where $f_{0}$ is the $3-\mathrm{dB}$ frequency of the white LED. The low-pass channel model given in (23) will be compared with the measured frequency response of the LUXEON3020 LED and then used in the theoretical calculations for bit rate and channel capacity.

\subsection{Noise variance analysis in DMT}

In VLC, each element in the noise vector is the sum of several noise components that degrade the channel capacity. The most two known components are the received thermal and the ambient shot light noises. Thermal noise is mostly generated by the photodetector (PD) and trans-impedance amplifier (TIA) and refers to as noise introduced by 
random, temperature dependent, and agitation of charge carried in the receiver electronics. On the other hand, shot noise is a result of an ambient light originating from sources such as sunlight, non-data carrying luminaries, and the mean intensity of the data carrying LED luminary itself. For a PIN/FET trans-impedance receiver, the thermal and shot noise variances are given by [49]:

$$
\begin{gathered}
\sigma_{\text {Thermal }}^{2}=\frac{8 \pi k T_{k}}{G_{o}} C_{p d} A_{r} I_{2} B^{2}+\frac{16 \pi^{2} k T_{k} \mu}{g_{m}} C_{p d}^{2} A_{r}^{2} I_{3} B^{3} \\
\sigma_{\text {Shot }}^{2}=2 q R P_{r} B+2 q I_{b g} I_{2} B
\end{gathered}
$$

where $k$ is the Boltzmann's constant, $T_{k}$ is the absolute temperature, $G_{o}$ is the open-loop voltage gain, $C_{p d}$ is the fixed capacitance of photo-detector per unit area, $\mu$ is the FET (field effect transistor) channel noise factor, $I_{2}$ and $I_{3}$ are the noise-bandwidth factors, $B$ is the bandwidth, $g_{m}$ is the FET transconductance, $q$ is the electron charge, and $I_{b g}$ is the photocurrent in the photo-diode caused by ambient light [50]. Generally, when considering indoor VLC systems and in the presence of intense background light, shot noise tends to be dominant due the high luminance requirements outlined in standards [36]. For typical luminance levels, the thermal noise could be neglected with respect to the shot noise level in the receiver.

However, when using DMT modulation over VLC, there are other factors that degrade the signal such as the nonlinearity and clipping distortions. DMT has typically a high PAPR which makes it very sensitive to nonlinear distortions caused generally by white LEDs. The distortion due to LED nonlinearity is usually related to the working current and the used range. Moreover, due to the finite dynamic range of digital to analog converters, clipping is applied to shape the signal with the desired amplitude range. The signal that exceeds a desired level $A_{c}$ is clipped. After clipping, the power distribution of the DMT signal is modified but at the price of clipping distortion. The noise variance $\sigma_{c}^{2}$ due to clipping can be calculated as follows [51, 52]:

$$
\sigma_{c}^{2}=\sigma_{s}^{2}\left[1-\operatorname{erfc}\left(\frac{\mu}{\sqrt{2}}\right)-\sqrt{\frac{2}{\pi}} \mu \exp \left(-\frac{\mu^{2}}{2}\right)-\left(1-\mu^{2}\right) \operatorname{erfc}\left(\frac{\mu}{\sqrt{2}}\right)\right]
$$

where $\sigma_{s}^{2}$ is the DMT symbols variance, $\mu=A_{c} / \sigma_{s}$, and erfc is the complementary error function [53].

Consequently, we can notice that the received signal in DMT transmission over VLCsuffers from mixed noise components arriving from different heterogeneous factors some of them being nonlinear. Moreover, we noticed from the theoretical analysis of the transmission bit rate of DMT with bit-loading that there are some parameters that must be carefully chosen to enhance the system performance.

In the next parts of this paper, we perform a global theoretical and experimental optimization of DMT-based VLC system parameters for real indoor applications. As the luminance is the most pertinent design parameter in VLC, looking for simplicity, we use only one white LED and adjust the distance between this LED and the receiver to ensure the required illumination level. A VLC transmission over link of several meters can be realized by using luminary consisting of $N_{L E D}$ white LEDs which number is defined according to (3), while keeping the standard illumination level as described in Section 2.1. 


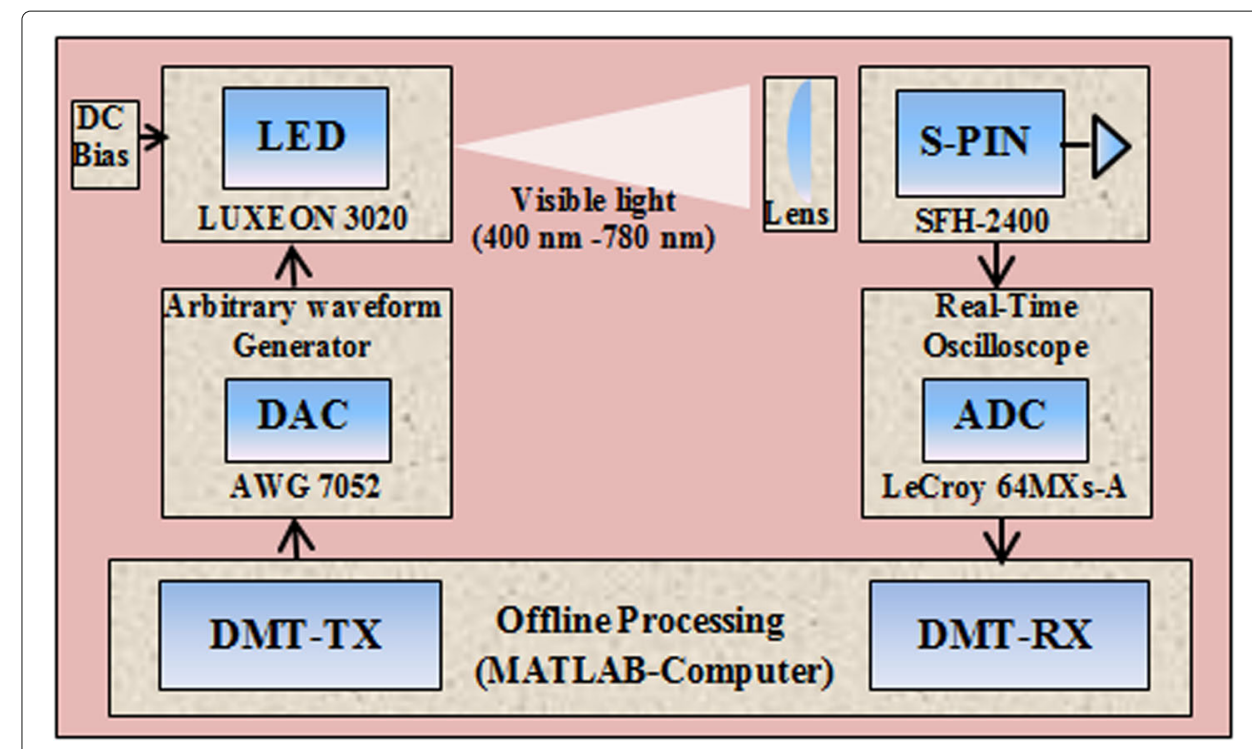

Fig. 4 Experimental setup of an indoor IM/DD VLC transmission

\section{Experimental setup}

The diagram and a photograph of our experimental setup are respectively shown in Figs. 4 and 5.

A commercially low-cost phosphorescent white light LUXEON3020 LED (L1303080002011001) is used as light source at the transmitter. The optical signal of LED is modulated by the electrical signal corresponding to the DMT symbols pre-generated in computer and stored in an arbitrary waveform generator (AWG7052TM, Tektronix, 10 bits resolution) memory. A lux-meter (LX-1010B) is used for setting the desired luminance level and adjust the distance between the transmitter and receiver. Using this device, a distance of approximately $20 \mathrm{~cm}$ is fixed between the LED and the receiver to

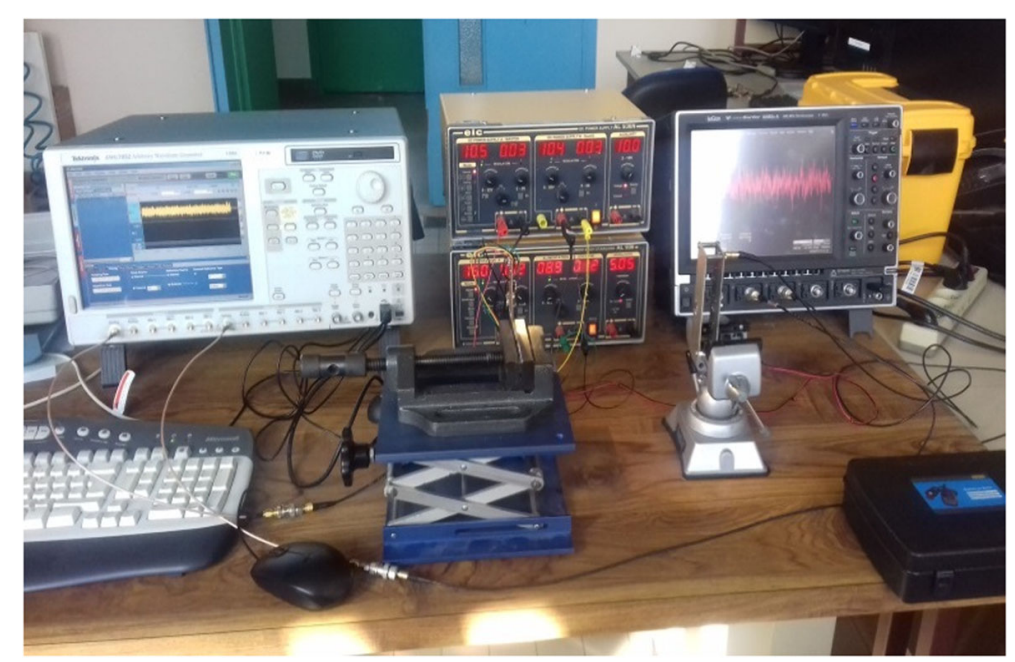

Fig. 5 Photograph of the experimental setup 
ensure 500 lux at the receiver side as recommended in DIN standards for working environment. This measured value is very close to the theoretical distance calculated owing to (2). Moreover, a network analyzer (HP-4195A) is utilized to measure the frequency response of the VLC link. The emitted white light from the LED is captured by a lens of a 16-mm diameter which is placed in front of the receiver in order to focus the emitted light onto a low-cost Silicon PIN (S-PIN) photo-detector (SFH-2400) with an active area of $1 \mathrm{~mm}^{2}$, a detection wavelength range from 350 to $1100 \mathrm{~nm}$, and a maximum responsivity of $0.65 \mathrm{~A} / \mathrm{W}$ at $870 \mathrm{~nm}$. A 50-MHz cut-off bandwidth TIA amplifier with trans-impedance gain of $110 \mathrm{k} \Omega$ is employed to amplify the electrical signal that will be then acquired by a real-time oscilloscope (LeCroy 64MXs-A, 8 bits resolution). Finally, offline processing is carried out with MatLab.

\section{Measured system parameters for optimization}

As mentioned in the experimental setup, the main components of VLC system are the white LED that acts as a communication source and the S-PIN photo-diode with the TIA amplifier serving as receiving elements. The receiver used in our study has a good behavior to visible wavelength region and does not provide a significant constraint compared to white LED that is very sensitive to the high frequencies representing a major challenge for high-data rate communications. Therefore, we measure in this section the most important communication features of the LUXEON3020 LED in terms of the frequency response, the time response, and the nonlinearity behavior.

\subsection{Frequency response of LED}

The frequency response of the VLC channel is an important factor especially for the bit allocation in DMT transmission employing bit-loading algorithms. The network analyzer is used to measure the frequency response of the VLC link at the output of the receiver and the measured results are shown in Fig. 6.

The measured frequency response shows a low-pass characteristic with a 3-dB bandwidth of $1.47 \mathrm{MHz}$. We traced in the same figure, the transfer function of the low-pass filter given by (23) for $f_{0}=1.47 \mathrm{MHz}$. From this figure, we can see that the transfer function of the low-pass filter is similar to the distribution of the measured frequency response, and it matches that of the LED in low frequencies range. Therefore, the transfer function given in (23) could be used as channel model for theoretical optimization. For a given illumination level, an optimum subcarrier number and a best modulated bandwidth will be determined in Section 6 .

\subsection{Time response of LED}

The time response of the VLC channel is crucial to calculate the cyclic prefix length. To measure its value, a pulse of $10 \mathrm{~ns}$ is generated by the AWG generator and sent via the LED. The received signal captured by the oscilloscope is shown in Fig. 7.

Measured results show that the maximum delay spread is around $450 \mathrm{~ns}$. So, the CP length of DMT symbols should be typically more than $450 \mathrm{~ns}$. Nevertheless, the major power of interference has delay spread less than $250 \mathrm{~ns}$. So, with the CP cost consideration, the CP length of each DMT symbol could be selected between 250 ns and 450 ns in order to produce the trade-off between the minimal inter-symbol interference and 


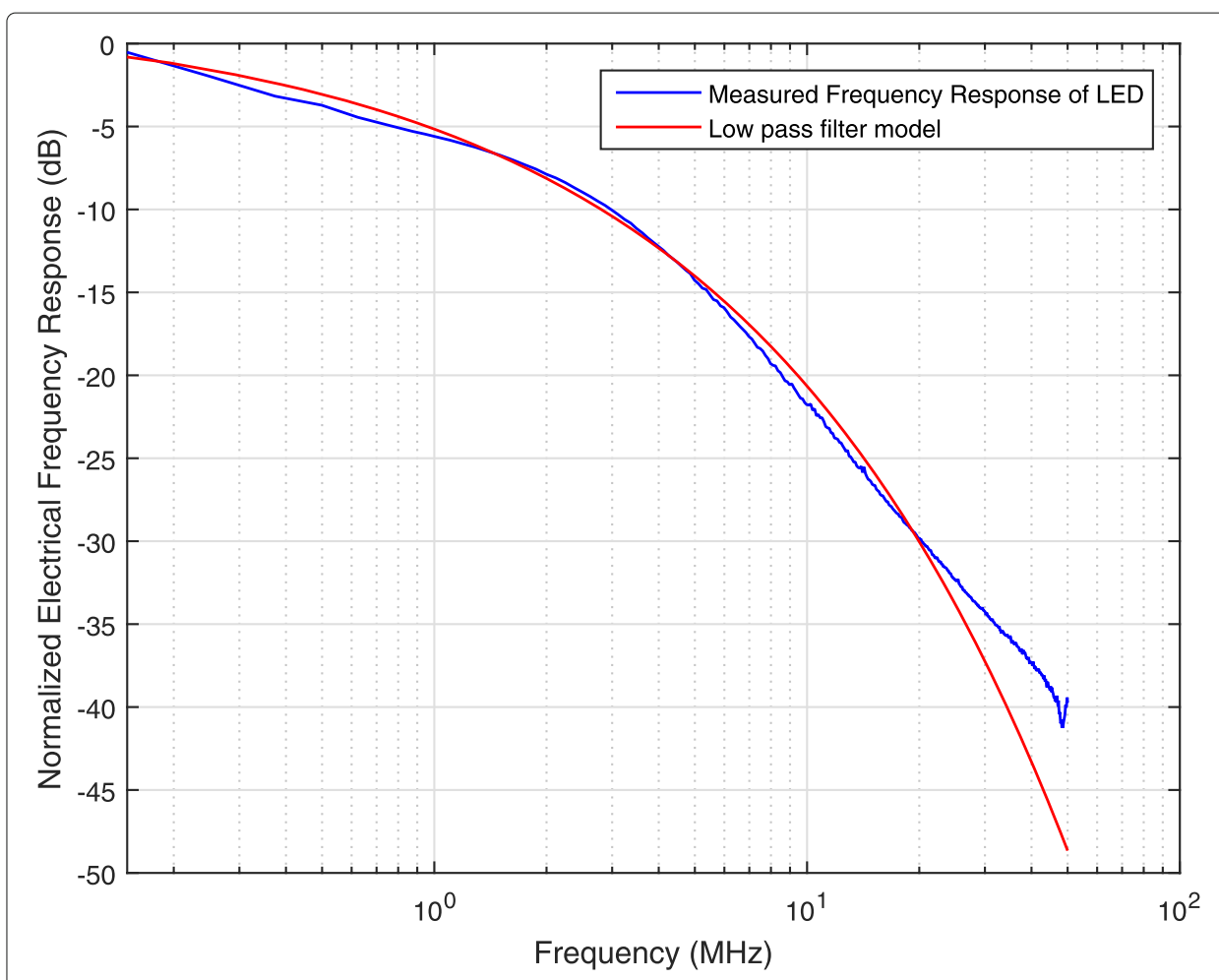

Fig. 6 Frequency response of LED. The frequency response measured by network analyzer at the output of the receiver

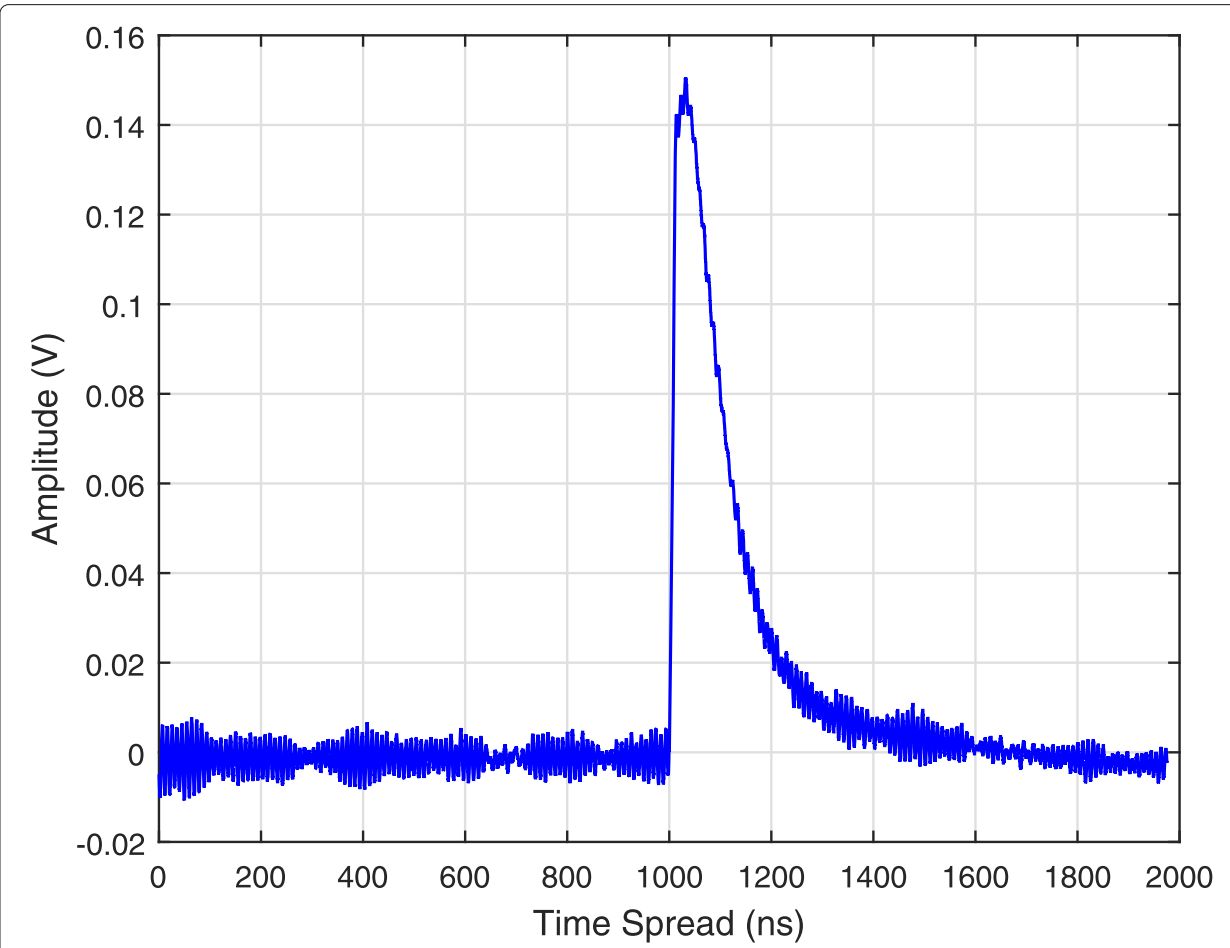

Fig. 7 Time response of LED. The signal captured by the oscilloscope for a pulse of 10 ns generated by AWG and sent via the LED 
the maximum spectral efficiency of DMT modulation. An optimization is experimentally performed in Section 7.3 to determine the best $C P$ length value.

\subsection{Nonlinearity behavior of LED}

Nonlinear distortion in VLC is mostly generated by the white LEDs. LEDs used for illumination are ideally driven by a DC current whose value is set to obtain the desired luminance level at a given surface. These devices should be operated within the allowed DC current limit indicated in the LED data sheet in order to avoid reduction in electrooptical efficiency due to the temperature instability [16]. In our work, this value is set to $120 \mathrm{~mA}$ under driving voltage of $3.05 \mathrm{~V}$. However, when the driving current is modulated, there is typically a variation in luminance and consequently in the radiated optical power of the LED according to the forward drive current. In the case of LUXEON3020 LEDs, the variation curve of optical power versus forward current is shown in Fig. 8 and could be formulated as:

$$
P_{\text {opt }}(I)=-0.0014 I^{2}+1.5864 I+1.0799
$$

where $I$ is the current expressed in $\mathrm{mA}$ and $P_{\text {opt }}$ the optical power expressed in $\mathrm{mW}$. We can notice from Fig. 8 that the optical power radiated from the LED increases not perfectly in a linear manner with the forward current because the number of photons emitted by the LED is not exactly proportional to the number of injected electrons in its active region. The nonlinearity behavior of the LED causes amplitude and phase distortions which introduce power losses and interference on adjacent carriers, especially with high-order modulations. Consequently, a reduction of the overall system performance

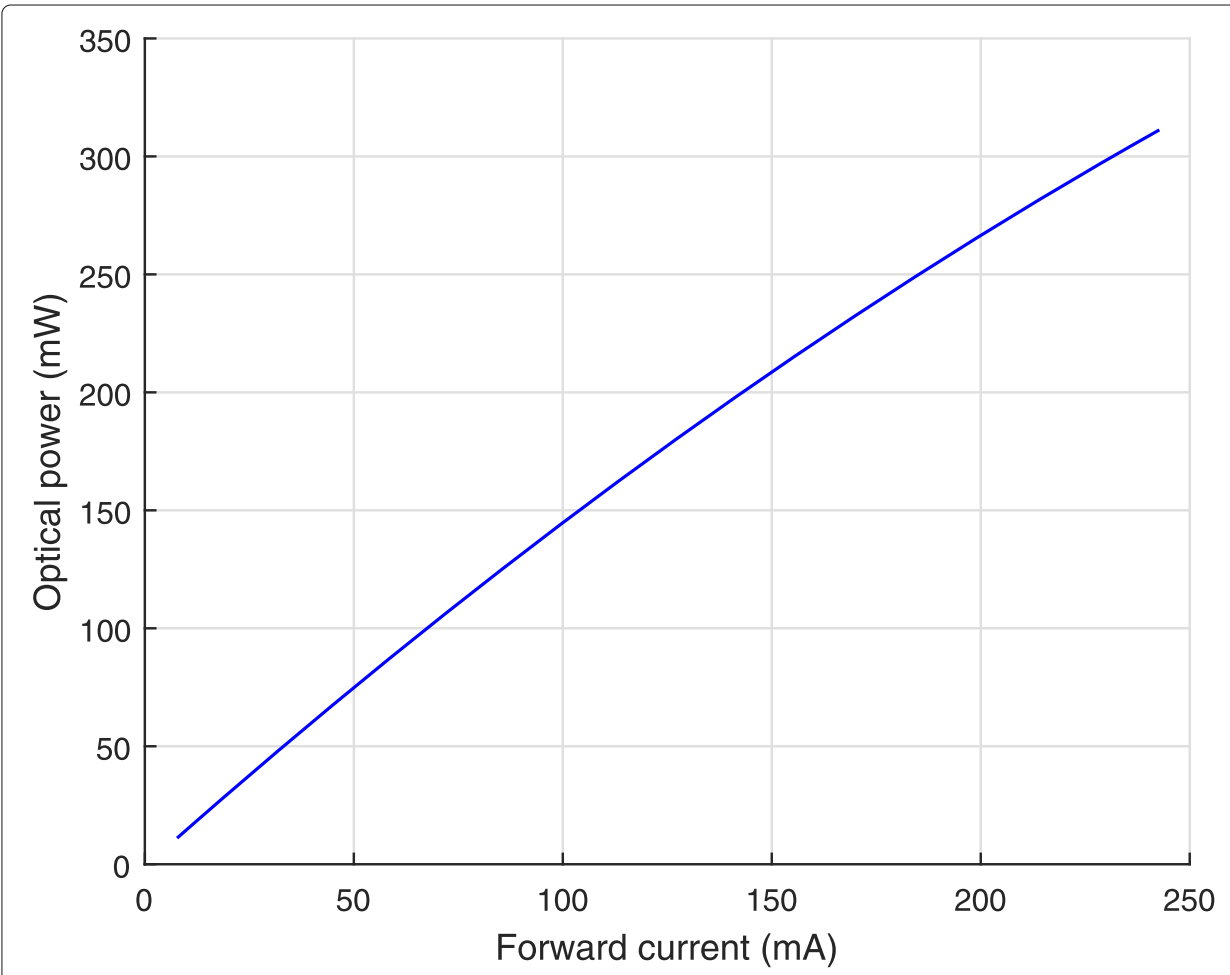

Fig. 8 Power transfer function of the LED. The variation curve of optical power versus forward current 
occurred. One method to counteract the nonlinearity effect is to operate the LED in a small dynamic range where its output characteristic is linear enough. However, the dispersive nature of non-coherent light and the resulting high path loss require the use of the greatest possible device active region [54]. Another method is to compensate the nonlinear characteristics of LED by digital pre-distortion which gives the optical power at the output of the LED the required shape.

If the current to optical power conversion were perfectly linear, with a $120-\mathrm{mA}$ biasing current, the maximum peak-to-peak range of signal current variation could be chosen from 0 to $240 \mathrm{~mA}$. However, since in practice, this function is not perfectly linear, this range is a bit lower. In our study, a digital pre-distortion function is used to linearize the dynamic range of the LED. After linearization, the current varies between 6 and $240 \mathrm{~mA}$, corresponding to an optical power between 11 and $302 \mathrm{~mW}$.

\section{Theoretical optimization results}

The goal of this section is to analyze the influence of bandwidth, subcarrier number, and luminance on the system performance. Based on Eqs. (21) and (22) and the channel model given in (23), we evaluate by carrying out simulations the transmission bit rate and the channel capacity for different values of these transmission parameters in order to determine their optimal values. For all these theoretical optimization results, we should notice that phase and clipping noises contribution have not been considered. Simulation parameters are listed in Table 1.

\subsection{Modulated bandwidth optimization}

The capacity and the transmission bit rate in a communication system are directly affected by the available bandwidth. Generally, the higher available bandwidth leads to better capacity. In the case of DMT modulation, the available bandwidth is typically divided into subcarriers and the total power are allocated to these subcarriers. Therefore, for a given luminance level, the higher modulated bandwidth value leads to lower allocated power to each subcarrier. So, a theoretical calculation of the channel capacity according to the used bandwidth is required. The spacing between subcarriers could be calculated by dividing the total used bandwidth into the subcarrier number as follows [22]:

$$
\Delta f=\frac{B}{N}
$$

We firstly have to determine $B_{\text {opt }}$ the optimal bandwidth size providing the maximal bit rate. For this purpose, we will compute the bit rate and the channel capacity for different

Table 1 Simulation parameters

\begin{tabular}{llll}
\hline & $\begin{array}{l}\text { Boptimization } \\
(6.1)\end{array}$ & Noptimization & $\begin{array}{l}\text { Capacity }=f\left(E_{h}\right) \\
(6.3)\end{array}$ \\
\hline Bandwidth & $B_{j}=j \Delta f$ & $B=28 \mathrm{MHz}$ & $B=28 \mathrm{MHz}$ \\
Subcarrier spacing $\Delta f$ & $15.625 \mathrm{kHz}$ & $B / \mathrm{N}_{j}$ & $54.6875 \mathrm{kHz}$ \\
Number of subcarriers & $64 \leq j \leq 8192$ & $16 \leq \mathrm{N}_{j}=2^{j} \leq 8192$ & 512 \\
$N_{0}$ & $-155 \mathrm{dBm} / \mathrm{Hz}$ & $-155 \mathrm{dBm} / \mathrm{Hz}$ & $-155 \mathrm{dBm} / \mathrm{Hz}$ \\
Luminance $E_{h}$ & $500 \mathrm{lux}$ & $500 \mathrm{lux}$ & $50 \leq E_{h} \leq 2500 \mathrm{lux}$ \\
$T_{s}$ & $64 \mu \mathrm{s}$ & $\frac{N_{j}}{B}$ & $18.28 \mu \mathrm{s}$ \\
\hline
\end{tabular}


bandwidths $B_{j}=j . \Delta f$ from $B_{\min }$ to $B_{\max }$ by correspondingly varying $j$. We chose $\Delta f$ to be quite smaller than the coherence bandwidth $\left(B_{c}\right)$ of the channel and to get the DMT symbol time duration $1 / \Delta f$ quite larger than the maximum delay spread $\tau_{\max }$.

According to these assumptions and based on (21), the capacity optimization problem can therefore be written as:

$$
\begin{aligned}
& \underset{j}{\operatorname{maximize}} C_{j}=\Delta f \cdot \sum_{i=1}^{j-1} \log _{2}\left(1+\frac{\left(R_{i}\left|H_{i}\right| A_{r} T_{s}(\psi) g(\psi) E_{h}\right)^{2}}{j^{2} \eta^{2} N_{0} \Delta f}\right) \\
& \text { subject to } B_{\min } \leq B_{j} \leq B_{\max } \text { with } B_{j}=j \Delta f \\
& \text { and } \quad \Delta f \text { is a constant such that } \Delta f<B_{c} \text { and } \frac{1}{\Delta f}>>\tau_{\max }
\end{aligned}
$$

so that according to Eq. (28), $B_{o p t}=N \Delta f$ where $N=\underset{j}{\arg \max } C_{j}$.

And according to (22), the bit rate optimization problem can be written as:

$$
\begin{array}{ll}
\underset{j}{\operatorname{maximize}} & D_{j}=\Delta f \cdot \sum_{i=1}^{j-1} \log _{2}\left(1+\frac{\left(R_{i}\left|H_{i}\right| A_{r} T_{s}(\psi) g(\psi) E_{h}\right)^{2}}{\Gamma j^{2} \eta^{2} N_{0} \Delta f}\right) \\
\text { subject to } & B_{\min } \leq B_{j} \leq B_{\max } \text { with } B_{j}=j \Delta f \\
\text { and } \quad \Delta f \text { is a constant such that } \Delta f<B_{c} \text { and } \frac{1}{\Delta f}>>\tau_{\max }
\end{array}
$$

From results shown in Fig. 7, the maximum delay spread can be approximated by $450 \mathrm{~ns}$, and therefore, $1 / \tau_{\max }=2.2 \mathrm{MHz}$ can be assumed as coherence bandwidth. For simulation, we chose $\Delta f=15.625 \mathrm{kHz}$ and modify the modulated bandwidth from 1 to $128 \mathrm{MHz}$. Consequently, the DMT symbol time duration $(64 \mu \mathrm{s})$ is constant for all tested bandwidths and is much higher than $\tau_{\max }$. For $B_{\min }=1 \mathrm{MHz}$ and $B_{\max }=128 \mathrm{MHz}$, the number of subcarriers correspondingly varies from 64 to 8192.

The noise power spectral density $N_{0}$ measured by the spectrum analyzer at the output of the receiver is $-155 \mathrm{dBm} / \mathrm{Hz}$. We suppose that the same luminance level (500 lux) is available in all simulation schemes. Using measured noise density $N_{0}$ and the channel model given in (23), the capacity and the transmission bit rate for a bit error rate of $10^{-3}$ according to the modulated bandwidth are shown in Fig. 9.

We can notice from Fig. 9 that a maximum system capacity or transmission bit rate could be reached for determined bandwidth values. As expected and described in [55], these values are much larger than the 3-dB bandwidth of the LUXEON3020 LED that is equal to $1.47 \mathrm{MHz}$. For the considered low-cost materials, the best system performance is obtained in the range between $20 \mathrm{MHz}$ and $40 \mathrm{MHz}$, and a maximal transmission bit rate of around $138 \mathrm{Mbps}$ could be obtained for bandwidth of $28 \mathrm{MHz}$.

\subsection{Subcarrier number optimization}

Having estimated the value of the optimal bandwidth that can be used to $B=28 \mathrm{MHz}$, we have now to estimate the optimal subcarrier number in this bandwidth in order to provide the highest bit rate. We therefore compute the bit rate for different number of subcarriers $N_{j}=2^{j}$ by varying $j$ for fixed $B$ and with time duration of cyclic prefix, $T_{C P}$, chosen equal to $450 \mathrm{~ns}$.

The problem can therefore be written as:

$$
\begin{aligned}
& \underset{j}{\operatorname{maximize}} D_{j}=\frac{1}{\left(N_{j} / B\right)+T_{C P}} \cdot \sum_{i=1}^{N_{j}-1} \log _{2}\left(1+\frac{\left(R_{i}\left|H_{i}\right| A_{r} T_{s}(\psi) g(\psi) E_{h}\right)^{2}}{\Gamma N_{j} \eta^{2} N_{0} B}\right) \\
& \text { subject to } N_{j}=2^{j} \\
& \text { and to } B=28 \mathrm{MHz} \text { and } T_{C P}=450 \mathrm{~ns}
\end{aligned}
$$




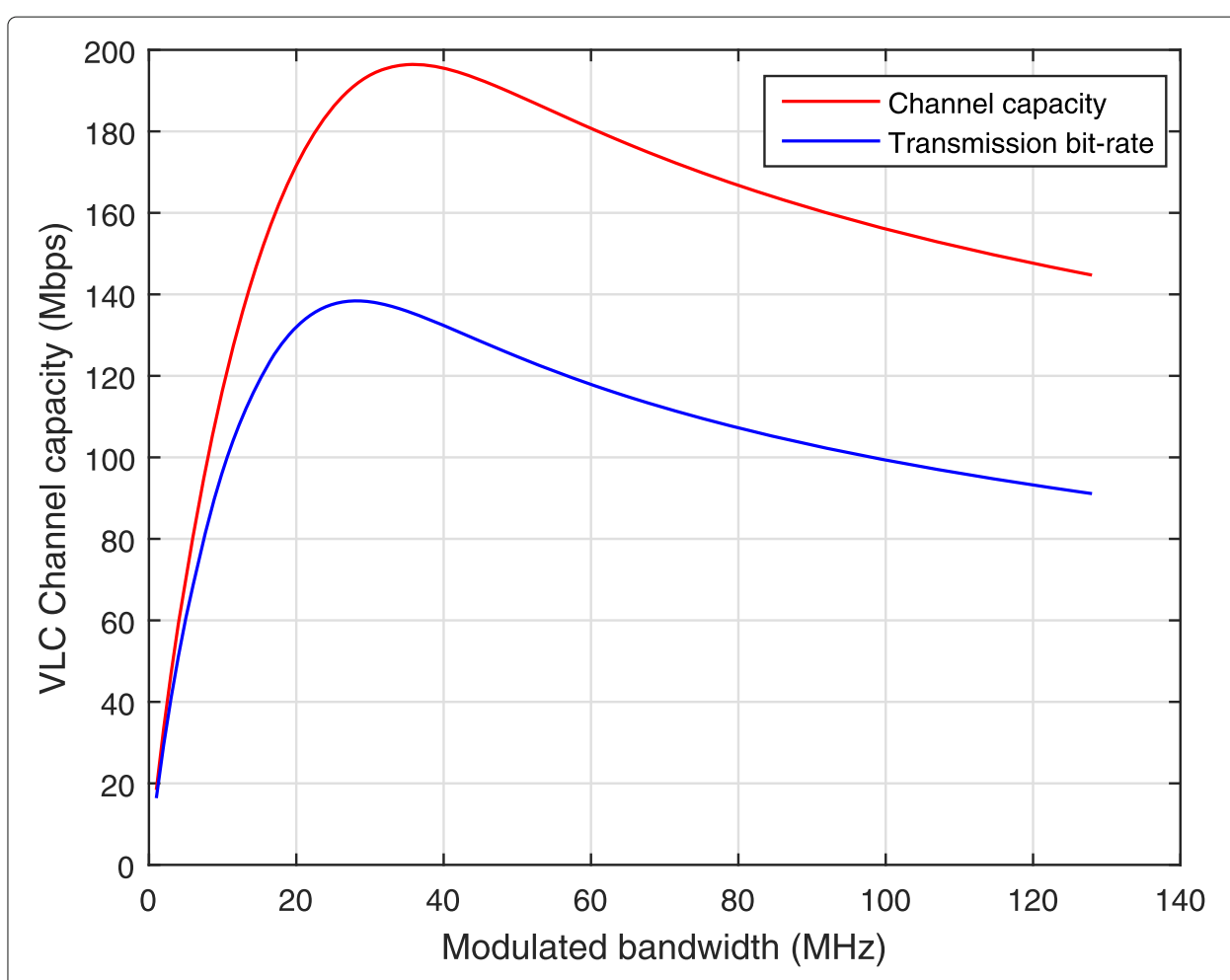

Fig. 9 System transmission bit rate with different bandwidths. The capacities and the transmission bit rates for a bit error rate of $10^{-3}$ according to the modulated bandwidth

so that according to (28), $\Delta f=\frac{B}{N}$ where $N=\underset{N_{j}}{\arg \max } D_{j}$.

For simulation, we modify the number of subcarriers from 16 to 8192 , and consequently, the subcarrier spacing $\Delta f$ varies from 1.75 to $0.0034 \mathrm{MHz}$. Again, we use the measured noise density $N_{0}$ and the channel model given in (23), to determine the maximum transmission bit rate according to the subcarrier number shown in Fig. 10.

We can see from the figure that the highest subcarrier number leads to the maximal transmission bit rates because of the $\mathrm{CP}$ length penalty. Consequently, smaller subcarrier spacing $(\Delta f)$ values lead to higher transmission bit rate. Moreover, we can observe that the transmission bit rate significantly increases for $N$ varying from 16 to 256 subcarriers, and a lower enhancement is occurred for $N$ higher than 512 subcarriers. Therefore, we consider in the rest of this paper $N=512$ subcarriers which offers a good compromise between the transmission data rate and the system complexity.

\subsection{Luminance level analysis}

As mentioned in DIN standard, a determined illumination level is assigned to each application in indoor working environments. Therefore and as VLC are designed to exploit illumination infrastructures for high speed communication, it is important to determine the relationship between the illumination level which is a useful metric for illumination design and the transmission bit rate that is an important parameter to evaluate a communication system. With the help of the optimized values of modulated bandwidth $(28 \mathrm{MHz})$ and subcarrier number (512), the transmission bit rates according to luminance level are depicted in Fig. 11. 


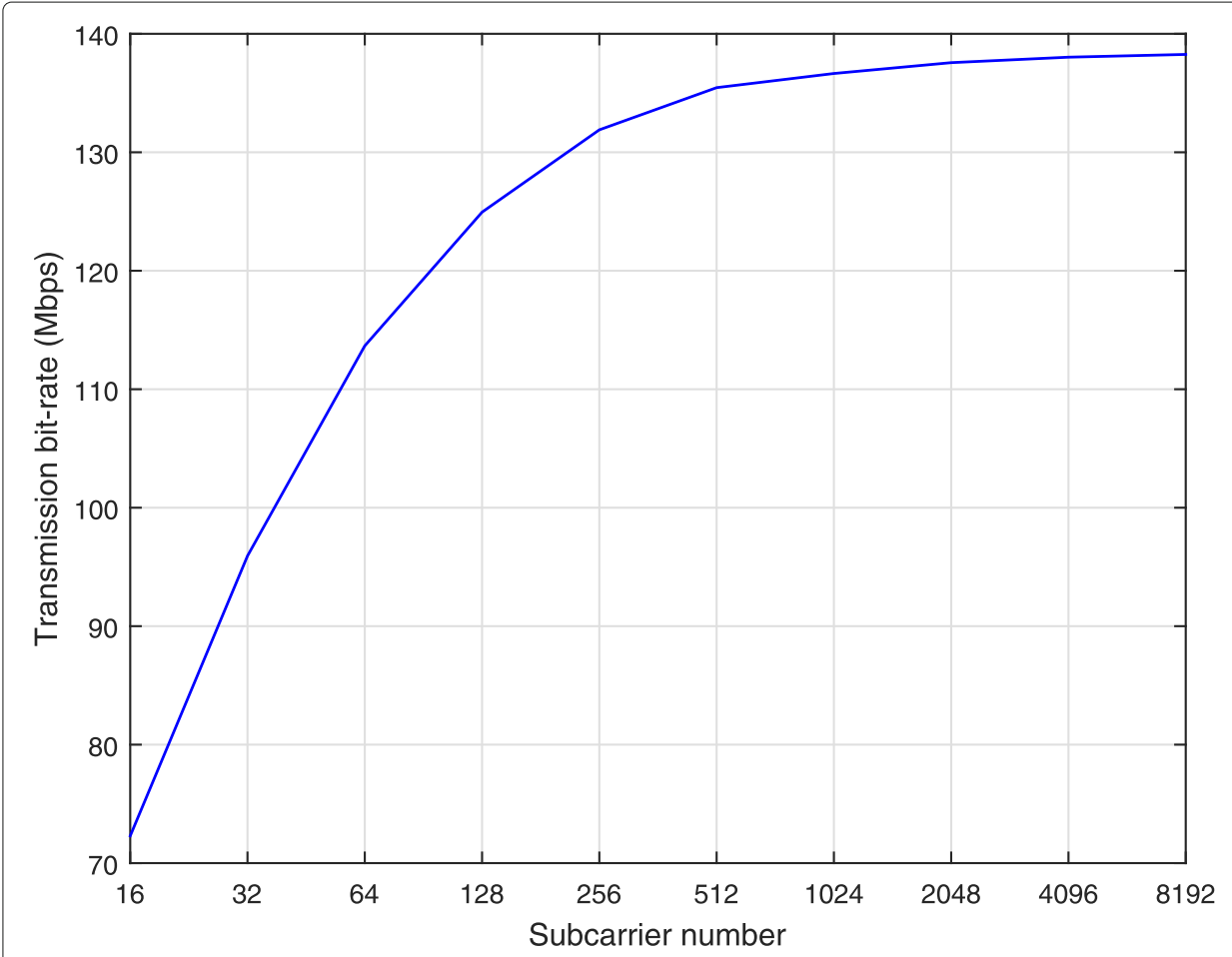

Fig. 10 System transmission bit rate with different subcarrier number

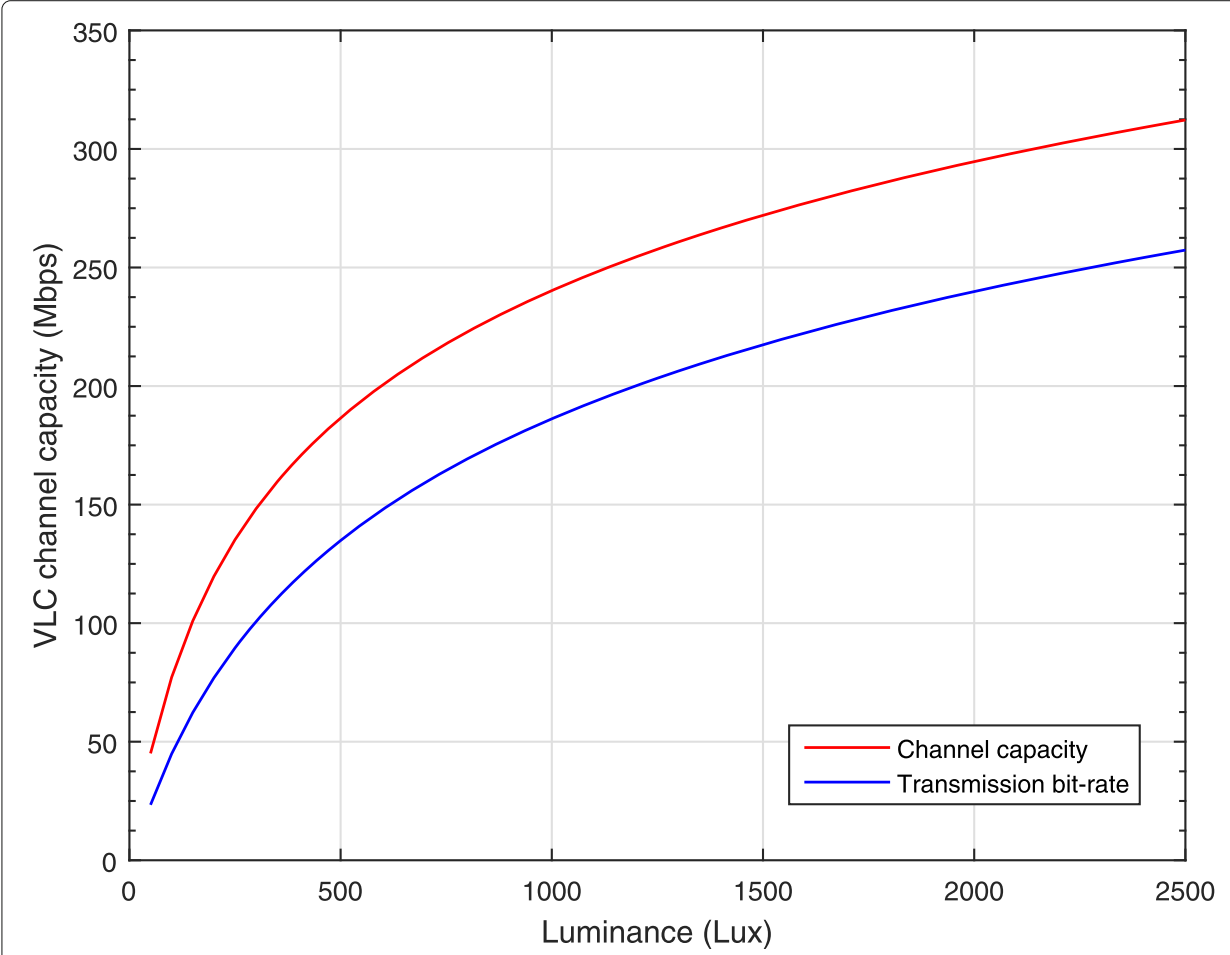

Fig. 11 Relationship between channel capacity and luminance 
We can see from Fig. 11 that the channel capacity and the bit rate in the VLC systems significantly increase with respect to luminance. Based on the required brightness level according to DIN standard and for the considered low-cost components, the maximal bitrate is about $138 \mathrm{Mbps}$ for luminance of $500 \mathrm{lux}$ (enough to read and write), $187 \mathrm{Mbps}$ for 1000 lux (well-lit office), and $240 \mathrm{Mbps}$ for 2000 lux (detailed drawing work). So, under the condition of a desired brightness level in front of the receiver, the maximum bit rates are different according to different applications.

\section{Searching optimal system setups}

In this section, experimental analysis are presented to determine the optimal configuration of DMT setups and verify the theoretical analysis presented in the previous section taking into account perturbations such as nonlinearity and phase noise. These first experimental results aim at defining the channel characteristics in terms of SNR per subcarrier in (7.1), the optimization of the clipping value in (7.2), the optimal bandwitdh used to provide the highest bit rate in (7.3), and the optimal value for the CP length in (7.4). We would remind that we use in this part a single LUXEON3020 LED that is biased at $120 \mathrm{~mA}$ and electrically driven by the sum of sinusoidal signals with different frequencies.

For all experiments, DMT signal are generated using MatLab and loaded into an AWG generator. The DMT symbol generation consists of bit generation, modulation symbol encoding, inverse fast Fourier transform, $\mathrm{CP}$ insertion, and clipping. After transmission through the visible light channel, the optical signal emitted by the LED is directly detected and converted to an electrical signal by the receiver that is situated in the front of the LED with distance of approximately $20 \mathrm{~cm}$, ensuring the direct line-of-sight (LOS) between transmitter-receiver and a luminance level of 500 lux. All experiments are performed at this brightness level checked by a lux-meter. The receiver is directly connected to the realtime oscilloscope. The oscilloscope is used for digitizing the signal from the TIA. Then, the samples are processed in MatLab performing down sampling, synchronization, FFT, one tape zero forcing equalization, and symbol de-mapping.

\subsection{Channel and SNR estimation}

For channel estimation, 30 DMT symbols are generated using MatLab with known QPSK modulation symbols and loaded into an AWG generator to be sent over the LED. Thanks to known QPSK symbols, the channel response is estimated and then the SNR level of each subcarrier is calculated and sent to the transmitter for being used by the bit allocation algorithm for next transmission of unknown data. BER is then calculated for each subcarrier for the $30 \mathrm{DMT}$ symbols, and the mean values are considered as the initial BERs. The channel information is derived by the corresponding analytical BER-vs-SNR expressions [56] and fed into the Chow's bit-loading algorithm [57] for throughput maximization. With a bit error rate of $10^{-3}$, the maximum transmission bit rate is finally calculated by (22).

\subsection{Experimental clipping optimization}

The high PAPR in DMT modulation leads to saturate the BER performance with higher order modulations. Reduction of the signal variance to be limited within the LED and DAC ranges without clipping, leads to a large power penalty. Clipping technique is proposed as an important method to reduce PAPR, but at the cost of noise increasing and 
its value becomes dominant when excessive signal clipping is applied. Consequently, clipping level selection is required to determine the optimal signal variance range according to the clipping noise. The maximal bit rate according to different clipping values is given in Fig. 12. The AWG is fed with a signal made of 2048 samples corresponding to a DMT symbol of $N=512$ subcarriers and up-sampling factor of $p=2$ applied after the $2 \mathrm{~N}$ samples provided by $2 \mathrm{~N}$ IFFT (cf. Fig. 3). The first subcarrier is not modulated in order to eliminate the DC component. A cyclic prefix of 32 samples is introduced before each DMT symbol. So, 2080 samples are generated for each symbol DMT and CP. DAC and ADC work at $120 \mathrm{MS} / \mathrm{s}$ and $5 \mathrm{GS} / \mathrm{s}$, respectively.

From the measured results, we can notice that the best performances are obtained when clipping ratio is $12-13 \mathrm{~dB}$. The maximal transmission bit rate $98.7 \mathrm{Mbps}$ is achieved with around $12 \mathrm{~dB}$ clipping value.

\subsection{Experimental bandwidth optimization}

As the system performance in real applications is related to the experimental environments, we evaluate the maximal bit rate according to different modulated bandwidths. We use the same setup parameters in the above section and only vary the bandwidth size. The clipping ratio is set to $12 \mathrm{~dB}$. DMT symbols are stored in an AWG memory and then generated by DAC with different sampling rate. DMT symbols are made of 512 subcarriers within a modulated bandwidth of $2.5,6,10,15,20,25,30,35,40$, and $45 \mathrm{MHz}$, respectively. The measured transmission bit rates with respect to modulated bandwidth are shown in Fig. 13.

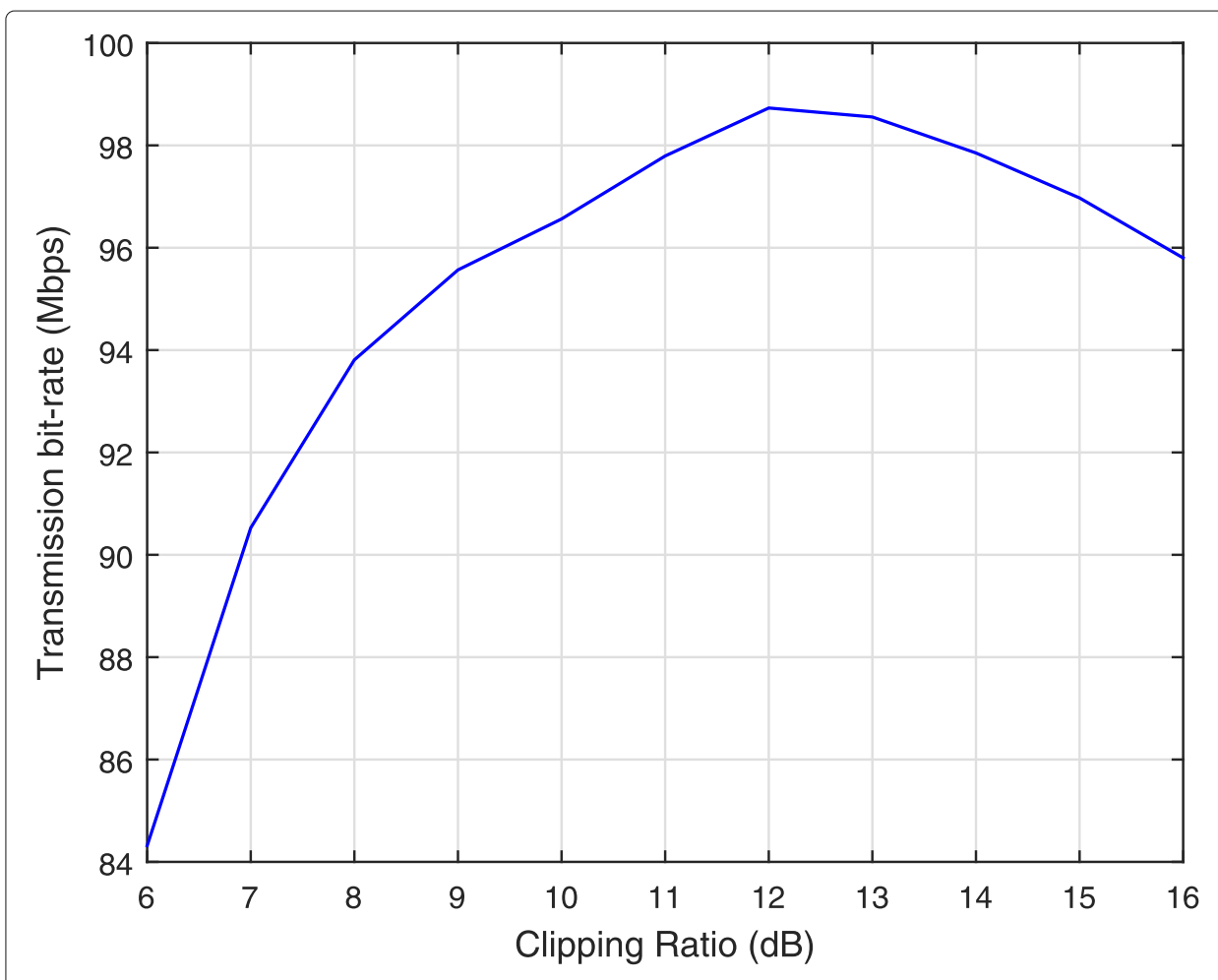

Fig. 12 Relationship between transmission bit rate and clipping level 


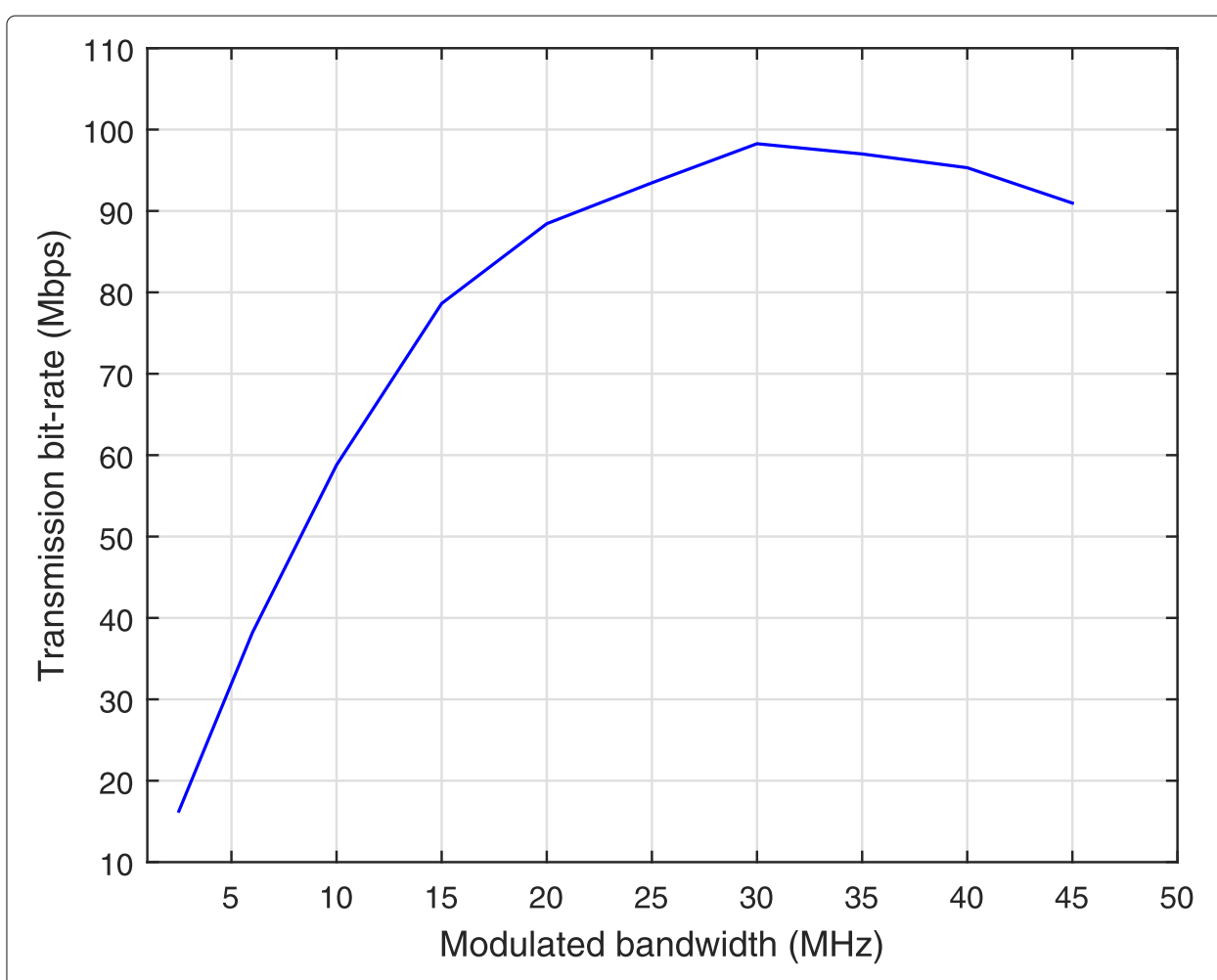

Fig. 13 Relationship between transmission bit rate and modulated bandwidth

As illustrated in the figure, the transmission bit rate significantly varies according to the modulated bandwidth variations. The best bit rates could be obtained when modulated bandwidth is between $25 \mathrm{MHz}$ and $40 \mathrm{MHz}$. The maximum value is achieved with $30 \mathrm{MHz}$, and the bit rate is around $99 \mathrm{Mbps}$. Moreover, compared to the theoretical results shown in Fig. 9, we can notice that approximately the same range of best modulated bandwidths is obtained by the theoretical and experimental results. The difference between the transmission bit rates is mainly caused by the divergence of the theoretical channel model from the real VLC channel and the limitation of experimental components, especially the practical ADC and DAC resolutions leading to significant increase of the quantization noise [58].

\subsection{Experimental CP length optimization}

With the help of the measured optimal bandwidth and clipping, we generate $30 \mathrm{MHz}$ DMT symbols with different CP length. A comparison of bit rates according to different $\mathrm{CP}$ lengths with and without the consideration of $\mathrm{CP}$ cost is depicted in Fig. 14. As 512 subcarriers are generated with 2048 samples and sending on $30 \mathrm{MHz}$ bandwidth, the DMT symbol time duration before the CP introduction is $17.0667 \mu$ and the sampling time interval is equal to $8.33 \mathrm{~ns}$.

As illustrated in the figure, the net transmission bit rate clearly enhances with the $\mathrm{CP}$ length. A significant improvement occurs when CP length is longer than 32 samples, and an important part of the interference power is removed at this value. Nevertheless, with the consideration of $\mathrm{CP}$ cost, we can notice that using a CP longer than 48 samples 


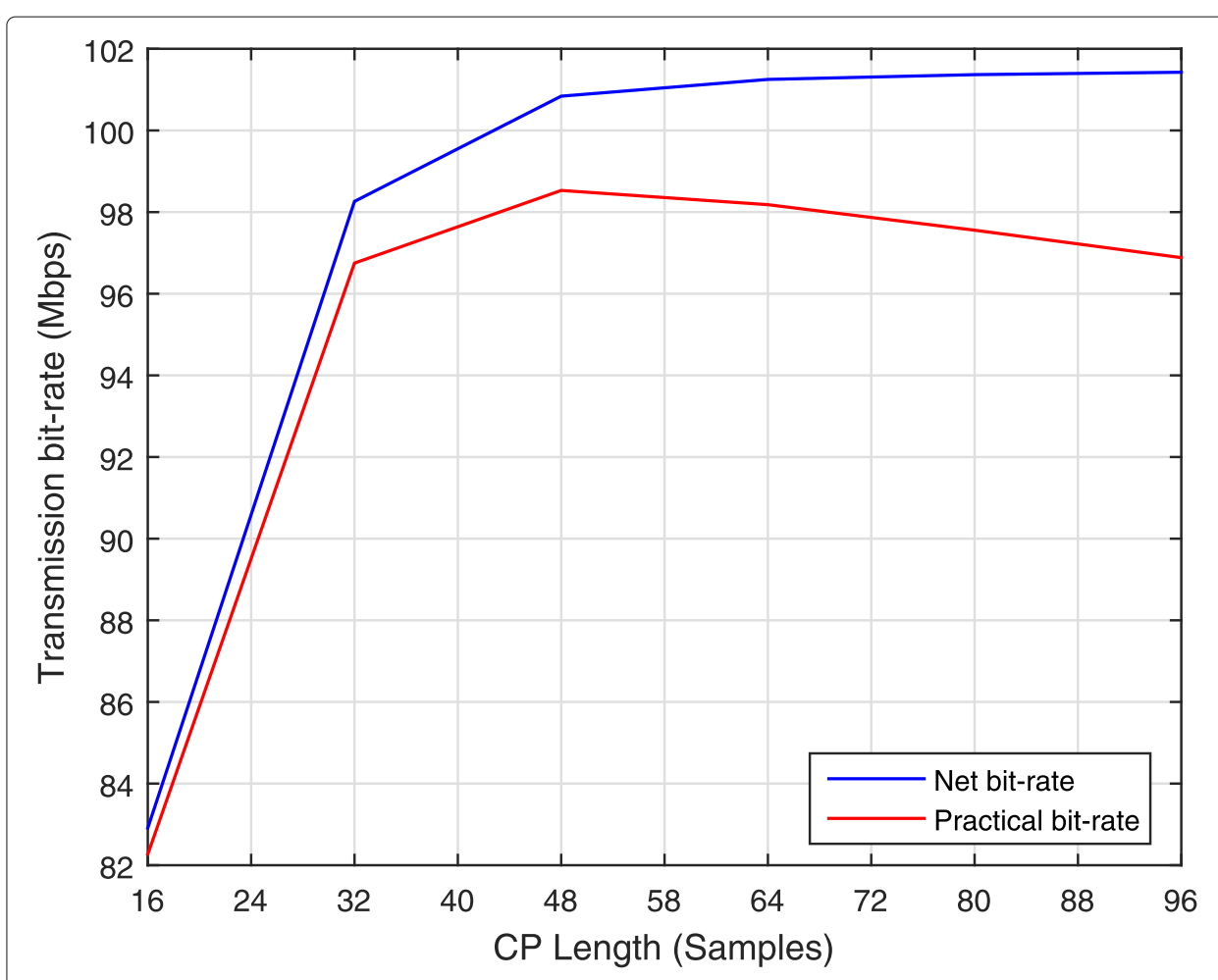

Fig. 14 Relationship between transmission bit rate and CP length

degrades the system performance. Beyond 48 samples, treat residual ISI is not compensated by the penalty introduced by the CP length increase. Consequently, 48 samples is the optimum $\mathrm{CP}$ length regarding to the performance contribution and $\mathrm{CP}$ length penalty. On the other hand, when CP length is between 32 and 48 samples, the CP time duration is between $266 \mathrm{~ns}$ and $400 \mathrm{~ns}$. These time values are clearly compatible with the results given in Section 5.2 and confirm the channel delay spread measurements illustrated in Fig. 7.

\section{100-Mbps transmission with optimal setup}

Finally, we generate DMT symbols with the optimum parameters mentioned in the previous sections. According to the theoretical and experimental results, 1000 DMT symbols with 512 subcarriers and 12- $\mathrm{dB}$ clipping ratio are generated in MatLab and sent in $30 \mathrm{MHz}$ modulated bandwidth with the help of bit-loading Chow algorithm. A CP length of $400 \mathrm{~ns}$ is introduced before each DMT symbol. The DAC in the AWG generator operates at $120 \mathrm{MS} / \mathrm{s}$. The LED works with biasing current of $120 \mathrm{~mA}$, and the signal peak-to-peak amplitude generated by the AWG is adjusted to ensure the input current of the LED in the limit range indicated by the LED data sheet. The ADC of the oscilloscope works at $5 \mathrm{GS} / \mathrm{s}$. The mean electrical power after passing by the VLC link is $-4.77 \mathrm{dBm}$ at the output of the receiver. The received signal is analyzed by offline processing in MatLab.

In the first step, the DMT symbols are generated where all subcarriers are modulated by a QPSK modulation having equal power. After synchronization and equalization, the channel frequency and impulse responses are estimated at 500 lux as shown in Fig. 15. The frequency and impulse responses thus estimated are fully in accordance with those 

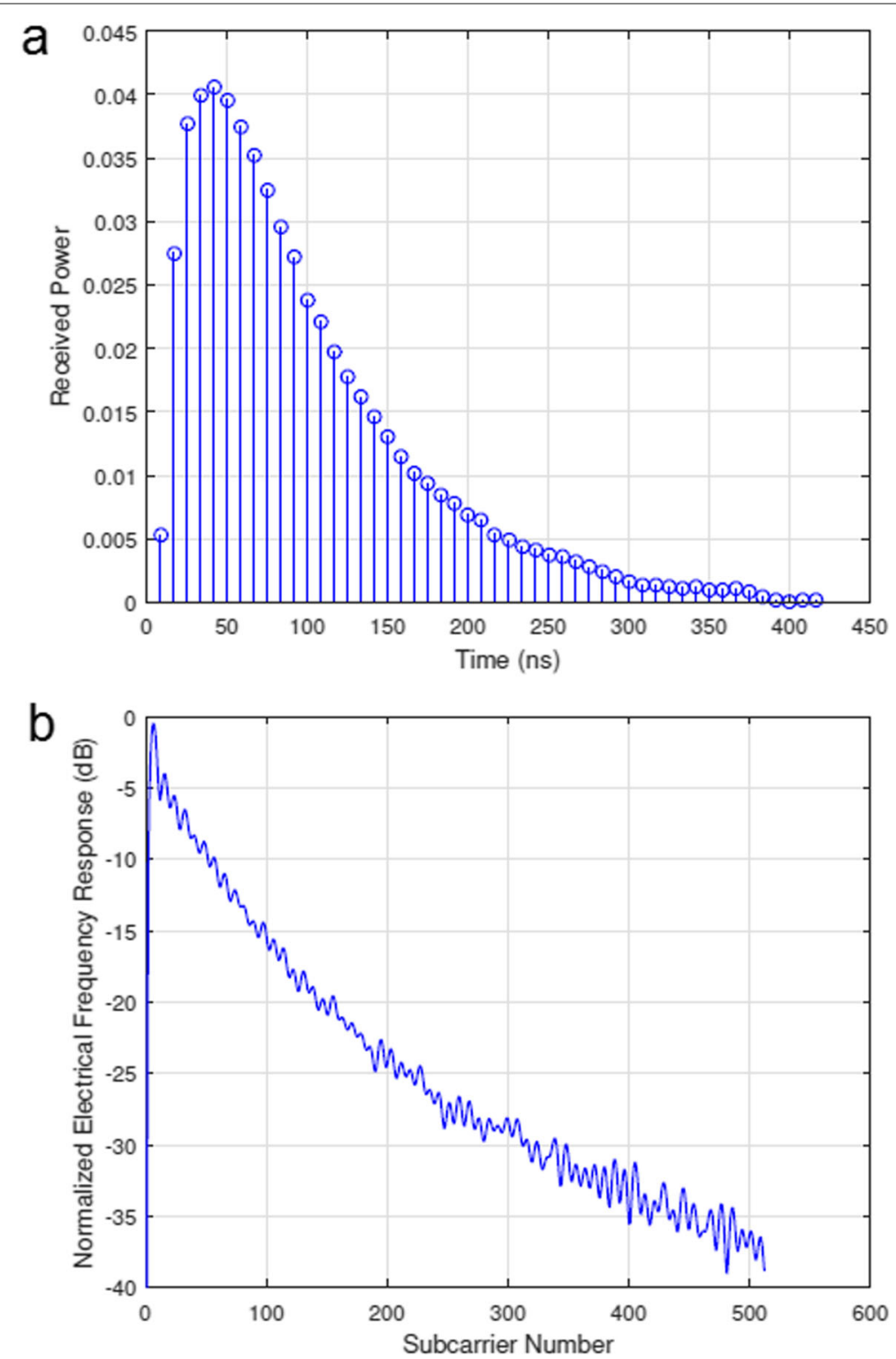

Fig. 15 Estimated $\mathbf{a}$ impulse and $\mathbf{b}$ frequency responses of the VLC channel at the output of the receiver

measured by using a network analyzer previously presented by Figs. 6 and 7. From the received DMT symbols, the SNR level of each subcarrier is calculated and fed into the Chow's bit-loading algorithm. According to different SNR values and for the target of $10^{-3}$ bit error rate, the corresponding allocated bits of each subcarrier are calculated. The SNR per subcarrier and corresponding allocated bits are depicted in Fig. 16.

In the next step, the DMT symbols are generated where subcarriers are modulated by different modulation orders as given by the bit-loading algorithm. Based on the Chow's bit-loading algorithm, 1 to 8 bits could be allocated to each subcarrier and modulation between 2-QAM and 256-QAM could be used in the effective modulation bandwidth. A total of 1721 bits can be transmitted over the 512 subcarriers leading to a transmission 


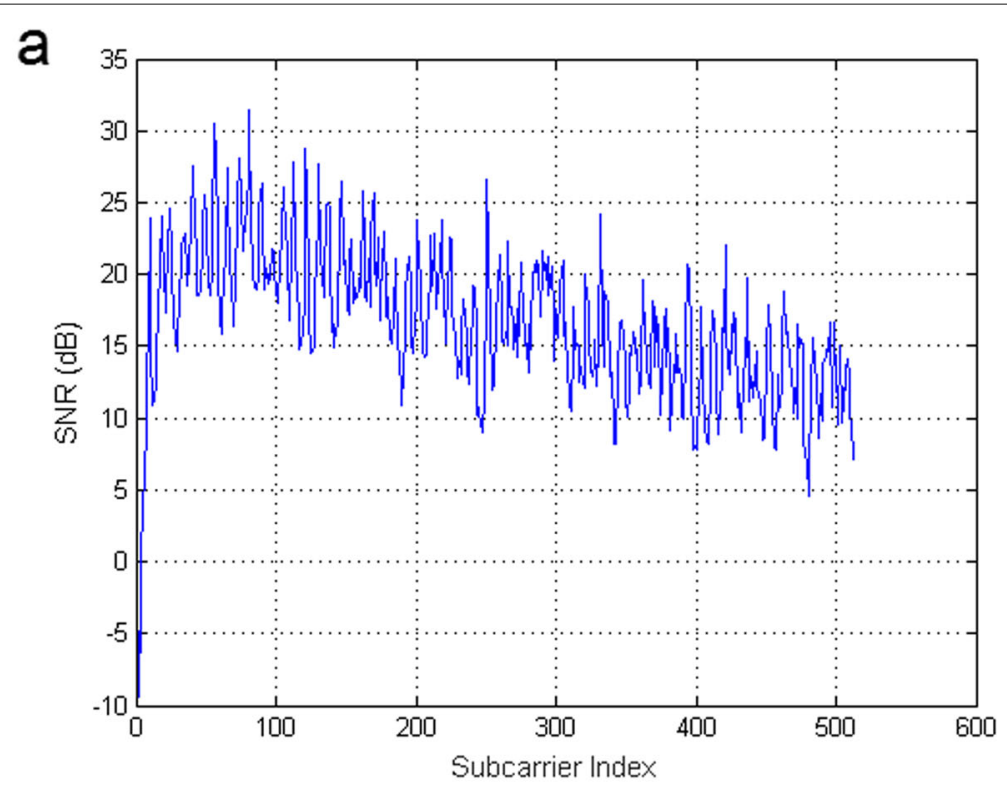

b

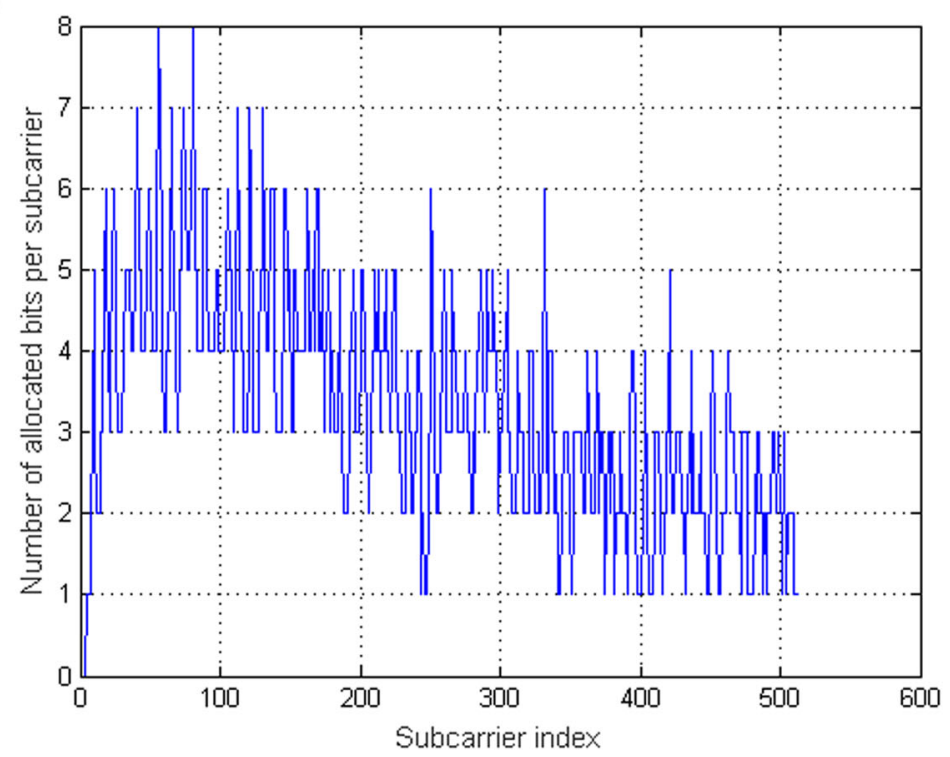

Fig. 16 SNR (a) and allocated bits (b) per subcarrier. Estimation per subcarrier at 500 lux

bit rate of 100.8 Mbps. The constellation maps of the 113th, 169th, 200th, and 405th subcarriers after equalization and demodulation are shown in Fig. 17. The mean BER in each subcarrier are traced in Fig. 18.

Finally and with the $\mathrm{CP}$ cost consideration, a practical transmission bit rate of $1721 \times$ $\left(120 \times 10^{6} \div(2048+48)\right) \approx 98.53 \mathrm{Mbps}$ is achieved at a total BER of $1.1 \times 10^{-3}$ which is very close to the target value.

\section{Conclusion}

In this paper, we proposed a global optimization of DMT transmission for VLC in indoor applications considering low-cost photo-detector and phosphor white LEDs used for simultaneous illumination and communication. After analytical description of typical 


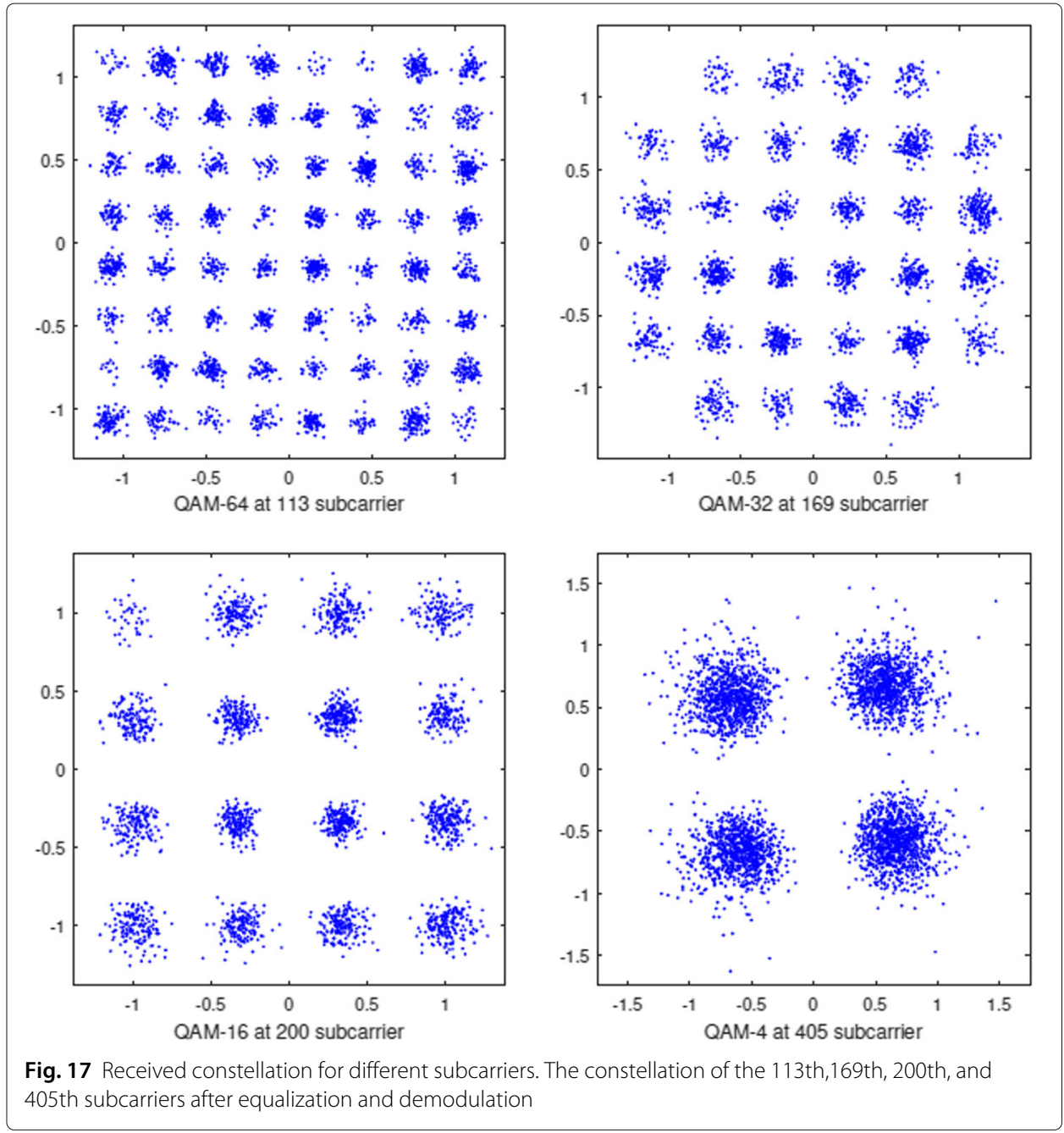

indoor lighting requirements, we investigated the capacity and the maximal bit rate of DMT transmission with bit-loading according to luminance levels. We illustrated that the system capacity in VLC significantly varies according to luminance, and consequently, the maximum bit rates are different according to each application. Moreover, based on the measured noise PSD and a proposed low-pass channel model similar to the measured real frequency response, we determined by theoretical calculation, the optimal subcarrier number, and bandwidth occupation. These results can be used to introduce the initial parameters for the practical DMT-based VLC transmission. Otherwise, in order to realize a complete optimization and as the system performance in real applications are related to the experimental environments, we analyzed and optimized the transmission bit rate according to different clipping levels by using experimental measurements. Experimental optimization is also made to validate the theoretical calculation of the modulated bandwidth as well as determine the best $\mathrm{CP}$ length. The selected value allows producing a trade-off between the minimal inter-symbol interference and the maximum spectral efficiency. Finally, based on the proposed configuration and with a regular luminance level for normal office work, we demonstrated a 100 -Mbps wireless VLC system with very 


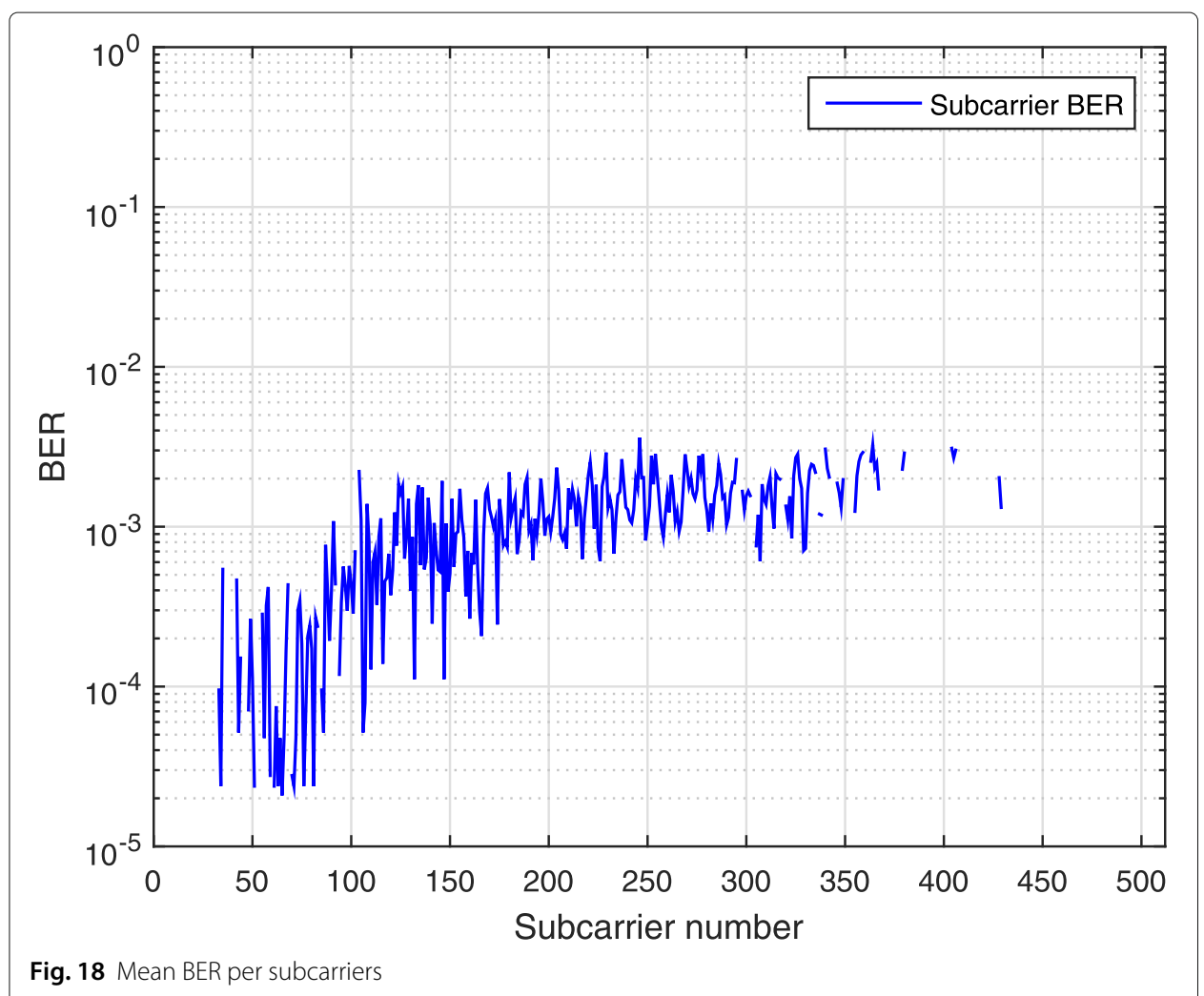

low-cost components. The analytical description as well as the optimization methodology presented in this paper are general and can be easily applied to other DMT- or OFDM -based VLC network configurations with different materials and components.

\section{Abbreviations}

VLC: Visible light communications; LED: Light-emitting diodes; DMT: Discrete multi-tone; IM/DD: Intensity modulation and direct detection; PD: Photo-detector; SNR: Signal-to-noise ratio; IFFT: Inverse fast Fourier transform; CP: Cyclic prefix; ISI: Inter-symbol interference; PAPR: Peak-to-average-power ratio; TIA: Trans-impedance amplifier; QAM : Quadrature amplitude modulation; BER: Bit-error rate.

\section{Acknowledgements}

Not applicable.

\section{Author's contributions}

AJ contributed to the entire studies of this work, manuscript preparation, and manuscript editing. SH contributed to the system design, experimental studies, and manuscript revision. $\mathrm{MH}$ proposed the conception of the optimization methods and contributed to the theoretical studies and manuscript revision. The authors read and approved the final manuscript.

\section{Funding}

This work was supported by the National Institute of Applied Sciences of Rennes.

\section{Availability of data and materials}

Not applicable.

\section{Competing interests}

The authors declare that they have no competing interests.

Received: 21 May 2019 Accepted: 5 April 2020

Published online: 14 May 2020

\section{References}

1. L. U. Khan, Visible light communication: applications, architecture, standardization and research challenges. Digit. Commun. Netw. 3(2), 78-88 (2017) 
2. Z. Ghassemlooy, S. Arnon, M. Uysal, Z. Xu, J. Cheng, Emerging optical wireless communications-advances and challenges. IEEE J. Sel. Areas Commun. 33(9), 1738-1749 (2015)

3. D. C. O'Brien, L. Zeng, H. Le-Minh, G. Faulkner, J. W. Walewski, S. Randel, in 2008 IEEE 19th International Symposium on Personal, Indoor and Mobile Radio Communications, Visible light communications: challenges and possibilities (IEEE, 2008). https://doi.org/10.1109/pimrc.2008.4699964

4. R. Ji, Q. Liu, W. Lu, S. Wang, High-speed visible light communications: enabling technologies and state of the art. Appl. Sci. 8, 1-19 (2018)

5. T. Fath, H. Haas, Performance comparison of MIMO techniques for optical wireless communications in indoor environments. IEEE Trans. Commun. 61(2), 733-742 (2013)

6. S. D. Lausnay, L. D. Strycker, J. Goemaere, B. Nauwelaers, N. Stevens, in 2013 2nd International Workshop on Optical Wireless Communications (IWOW), Design of a visible light communication transmitter for the evaluation of a wide range of modulation techniques (IEEE, 2013). https://doi.org/10.1109/iwow.2013.6777771

7. O. Bouchet, P. Porcon, M. Wolf, L. Grobe, J. W. Walewski, S. Nerreter, K. Langer, L. Fernández, J. Vucic, T. Kamalakis, G. Ntogari, E. Gueutier, in 2010 IEEE Globecom Workshops, Visible-light communication system enabling $73 \mathrm{Mb} / \mathrm{s}$ data streaming (IEEE, 2010). https://doi.org/10.1109/glocomw.2010.5700092

8. J.-Y. Sung, C.-W. Chow, C.-H. Yeh, Is blue optical filter necessary in high speed phosphor-based white light LED visible light communications?. Opt. Express. 22(17), 20646-20651 (2014)

9. H. Haas, L. Yin, Y. Wang, C. Chen, What is lifi?. J. Lightwave Technol. 34(6), 1533-1544 (2016)

10. H. Chun, S. Rajbhandari, G. Faulkner, D. O'Brien, in 2014 3rd International Workshop in Optical Wireless Communications (IWOW), Effectiveness of blue-filtering in WLED based indoor visible light communication (IEEE, 2014). https://doi. org/10.1109/iwow.2014.6950777

11. G. Stepniak, M. Schüppert, C. Bunge, Advanced modulation formats in phosphorous led VLC links and the impact of blue filtering. J. Lightwave Technol. 33(21), 4413-4423 (2015)

12. J. Vučić, L. Fernández, C. Kottke, K. Habel, K.-. Langer, in 36th European Conference and Exhibition on Optical Communication, Implementation of a real-time DMT-based 100 Mbit/s visible-light link (IEEE, 2010). https://doi.org/ $10.1109 /$ ecoc.2010.5621171

13. J. Vucic, C. Kottke, S. Nerreter, K. Langer, J. W. Walewski, 513 Mbit/s visible light communications link based on DMT-modulation of a white led. J. Lightwave Technol. 28(24), 3512-3518 (2010)

14. J. Vučić, C. Kottke, K. Habel, K. Langer, in 2011 Optical Fiber Communication Conference and Exposition and the National Fiber Optic Engineers Conference, $803 \mathrm{Mbit} / \mathrm{s}$ visible light WDM link based on DMT modulation of a single RGB led luminary (IEEE, 2011). https://doi.org/10.1364/ofc.2011.owb6

15. C. Kottke, J. Hilt, K. Habel, J. Vučić, K. Langer, in European Conference and Exhibition on Optical Communication, 1.25 Gbit/s visible light WDM link based on DMT modulation of a single RGB led luminary (OSA, 2012). https://doi.org/10. 1364/eceoc.2012.we.3.b.4

16. A. M. Khalid, G. Cossu, R. Corsini, P. Choudhury, E. Ciaramella, 1-gb/s transmission over a phosphorescent white LED by using rate-adaptive discrete multitone modulation. IEEE Photon. J. 4(5), 1465-1473 (2012)

17. G. Cossu, A. M. Khalid, P. Choudhury, R. Corsini, E. Ciaramella, $3.4 \mathrm{Gbit} / \mathrm{s}$ visible optical wireless transmission based on RGB led. Opt. Express. 20(26), 501-506 (2012)

18. J.-Y. Sung, C.-W. Chow, C.-H. Yeh, Dimming-discrete-multi-tone (DMT) for simultaneous color control and high speed visible light communication. Opt. Express. 22(7), 7538-7543 (2014)

19. Z. Zheng, L. Liu, W. Hu, Accuracy of ranging based on DMT visible light communication for indoor positioning. IEEE Photon. Technol. Lett. 29(8), 679-682 (2017)

20. Rakesh Krishna VS, I. Djordjevic, in Metro and Data Center Optical Networks and Short-Reach Links, DMT visible light communication using commercial RGBA leds (SPIE, the international society for optics and photonics, 2018), p. 18. https://doi.org/10.1117/12.2288784. https://spie.org/Publications/Proceedings/Paper/10.1117/12.2288784?SSO=1

21. L. Peng, S. Haese, M. Hélard, Optimized discrete multitone communication over polymer optical fiber. IEEE/OSA J. Opt. Commun. Netw. 5(11), 1313-1327 (2013)

22. L. Peng, M. Hélard, S. Haese, in 2013 IEEE International Conference on Communications (ICC), Optimization of multi-band DFT-spread DMT system for polymer optical fiber communications (IEEE, 2013). https://doi.org/10.1109/ icc.2013.6655159

23. P. Tian, J. J. D. McKendry, E. Gu, Z. Chen, Y. Sun, G. Zhang, M. D. Dawson, R. Liu, Fabrication, characterization and applications of flexible vertical InGan micro-light emitting diode arrays. Opt. Express. 24(1), 699-707 (2016)

24. R. X. G. Ferreira, E. Xie, J. J. D. McKendry, S. Rajbhandari, H. Chun, G. Faulkner, S. Watson, A. E. Kelly, E. Gu, R. V. Penty, I. H. White, D. C. O'Brien, M. D. Dawson, High bandwidth GaN-based micro-LEDs for multi-Gb/s visible light communications. IEEE Photon. Technol. Lett. 28(19), 2023-2026 (2016)

25. M. S. Islim, R. X. Ferreira, X. He, E. Xie, S. Videv, S. Viola, S. Watson, N. Bamiedakis, R. V. Penty, I. H. White, A. E. Kelly, E. Gu, H. Haas, M. D. Dawson, Towards 10Gb/s orthogonal frequency division multiplexing-based visible light communication using a GaN violet micro-LED. Photon. Res. 5(2), 35-43 (2017)

26. M. T. Sajjad, P. P. Manousiadis, H. Chun, D. A. Vithanage, S. Rajbhandari, A. L. Kanibolotsky, G. Faulkner, D. O'Brien, P. J. Skabara, I. D. W. Samuel, G. A. Turnbull, Novel fast color-converter for visible light communication using a blend of conjugated polymers. ACS Photon. 2(2), 194-199 (2015)

27. J. M. M. Santos, S. Rajbhandari, D. Tsonev, H. Chun, B. Guilhabert, A. B. Krysa, A. E. Kelly, H. Haas, D. C. O'Brien, N. Laurand, M. D. Dawson, Visible light communication using InGan optical sources with AllnGaP nanomembrane down-converters. Opt. Express. 24(9), 10020-10029 (2016)

28. I. Dursun, C. Shen, M. R. Parida, J. Pan, S. P. Sarmah, D. Priante, N. Alyami, J. Liu, M. I. Saidaminov, M. S. Alias, A. L. Abdelhady, T. K. Ng, O. F. Mohammed, B. S. Ooi, O. M. Bakr, Perovskite nanocrystals as a color converter for visible light communication. ACS Photon. 3(7), 1150-1156 (2016)

29. I. Lu, C. Yeh, D. Hsu, C. Chow, Utilization of 1-GHz VCSEL for 11.1-Gbps ofDM VLC wireless communication. IEEE Photon. J. 8(3), 1-6 (2016)

30. H.-H. Lu, C.-Y. Li, C.-A. Chu, T.-C. Lu, B.-R. Chen, C.-J. Wu, D.-H. Lin, 10 Gbps LiFi transmission system based on a two-stage injection-locked $680 \mathrm{~nm}$ VCSEL transmitter. Opt. Lett. 40(19), 4563-4566 (2015) 
31. T. Luan, K. Qian, Research on influencing factors of LED frequency response. AIP Conf. Proc. 1864(1), 1-8 (2017)

32. Z. Ghassemlooy, S. R. W. Popoola, CRC Press, Optical wireless communications: system and channel modelling with matlab, (Florida, 2013)

33. A. Sewaiwar, S. V. Tiwari, Y. H. Chung, Smart LED allocation scheme for efficient multiuser visible light communication networks. Opt. Express. 23(10), 13015-13024 (2015)

34. T.-C. Bui, S. Kiravittaya, K. Sripimanwat, N.-H. Nguyen, A comprehensive lighting configuration for efficient indoor visible light communication networks. Int. J. Opt. 2016, 1-9 (2016)

35. Lumileds, Data sheet LUXEON 3020. DS209-pdf (2017). https://www.lumileds.com/products/mid-power-leds/ luxeon-3020. Accessed 07 Mar 2017

36. E. Monteiro, S. Hranilovic, Design and implementation of color-shift keying for visible light communications. J. Lightwave Technol. 32(10), 2053-2060 (2014)

37. DIN, Std. 5035. Artif. Light. Inter. (1990). German Institute for Standardisation (Deutsches Institut für Normung)

38. M. S. A. Mossaad, S. Hranilovic, L. Lampe, Visible light communications using OFDM and multiple LEDs. IEEE Trans. Commun. 63(11), 4304-4313 (2015)

39. H. Elgala, R. Mesleh, H. Haas, Indoor broadcasting via white LEDs and OFDM. IEEE Trans. Consum. Electron. 55(3), 1127-1134 (2009)

40. CIE, The International Commission on Illumination. Light True Vis. Quant. Princ. Meas. (1979). ISBN: 9783900734473. http://cie.co.at/publications/light-true-visual-quantity-principles-measurement

41. K. Lee, H. Park, J. R. Barry, Indoor channel characteristics for visible light communications. IEEE Commun. Lett. 15(2), 217-219 (2011)

42. J. Grubor, S. Randel, K. Langer, J. W. Walewski, Broadband information broadcasting using LED-based interior lighting. J. Lightwave Techno. 26(24), 3883-3892 (2008)

43. L. Peng, S. Haese, M. Hélard, in 201219 th International Conference on Telecommunications (ICT), Optimum configuration for discrete multi-tone transmission over polymer optical fiber (IEEE, 2012). https://doi.org/10.1109/ ictel.2012.6221284

44. J. G. Proakis, M. Saheli, Digital communications, 5th ed. (McGraw-Hill Inc, New York, 2007)

45. B. Cardiff, M. F. Flanagan, F. Smyth, L. P. Barry, A. D. Fagan, On bit and power loading for OFDM over SI-POF. J. Lightwave Technol. 29(10), 1547-1554 (2011)

46. A. Garcia-Armada, SNR gap approximation for M-PSK-based bit loading. IEEE Trans. Wirel. Commun. 5(1), 57-60 (2006)

47. W. Yu, J. M. Cioffi, in ICC 2001. IEEE International Conference on Communications. Conference Record (Cat. No.01CH37240), On constant power water-filling, vol. 6, (2001), pp. 1665-1669

48. H. Haas, C. Chen, D. O'Brien, A guide to wireless networking by light. Prog. Quantum Electron. 55, 88-111 (2017)

49. W. Zhang, M. I. Sakib Chowdhury, M. Kavehrad, Asynchronous indoor positioning system based on visible light communications. Opt. Eng. 53, 53-5310 (2014)

50. T. Komine, M. Nakagawa, Fundamental analysis for visible-light communication system using LED lights. IEEE Trans. Consumer Electron. 50(1), 100-107 (2004)

51. S. Randel, F. Breyer, S. C. J. Lee, J. W. Walewski, Advanced modulation schemes for short-range optical communications. IEEE J. Sel. Topics Quant. Electron. 16(5), 1280-1289 (2010)

52. C. R. Berger, Y. Benlachtar, R. I. Killey, P. A. Milder, Theoretical and experimental evaluation of clipping and quantization noise for optical OFDM. Opt. Express. 19(18), 17713-17728 (2011)

53. A. Milton, S. I. A, Handbook of mathematical functions with formulas, graphs, and mathematical tables, p. 297. (Dover, New York, 1965)

54. H. Burchardt, N. Serafimovski, D. Tsonev, S. Videv, H. Haas, VLC: beyond point-to-point communication. IEEE Commun. Mag. 52(7), 98-105 (2014)

55. S. Dimitrov, W. Haas, Principles of LED light communications. (Cambridge University Press, Cambridge, 2015)

56. R. A. Shafik, M. S. Rahman, A. R. Islam, in 2006 International Conference on Electrical and Computer Engineering, On the extended relationships among EVM, BER and SNR as performance metrics (IEEE, 2006). https://doi.org/10.1109/icece. 2006.355657

57. P. S. Chow, J. M. Cioffi, J. A. C. Bingham, A practical discrete multitone transceiver loading algorithm for data transmission over spectrally shaped channels. IEEE Trans. Commun. 43(2/3/4), 773-775 (1995)

58. E. Chen, C. K. Yang, Adc-based serial i/o receivers. IEEE Trans. Circ. Syst. I: Regular Pap. 57(9), 2248-2258 (2010)

Publisher's Note

Springer Nature remains neutral with regard to jurisdictional claims in published maps and institutional affiliations. 